\title{
Fluid flow beneath a semipermeable membrane during drying processes
}

\author{
Maurice J. Blount, Michael J. Miksis, and Stephen H. Davis \\ Department of Engineering Sciences and Applied Mathematics, Northwestern University, Evanston, Illinois 60208, USA
}

(Received 12 September 2011; published 31 January 2012)

\begin{abstract}
The dynamic interactions between a semipermeable membrane and a long, thin layer of liquid beneath it are investigated in the context of drying processes. The membrane separates two aqueous solutions of sugar, and the transport of water across the membrane is driven by concentration and pressure gradients across it. A model is formulated using a long-wave approximation that includes the effects of volume loss due to water transport across the membrane, the incompressibility and bending stiffness of the membrane, and the dynamical effects that arise owing to the viscous stresses generated by the fluid flow. This model is first applied to study the desiccation of a sessile vesicle that is clamped to a rigid substrate and then also to study the behavior of blisters on laminated substrates. For each problem, equilibrium membrane shapes are obtained and their bifurcation structures are described as the sugar concentration above the membrane is varied. It is demonstrated that a wrinkled membrane coarsens to lessen the frequency of wrinkles and that if the membrane is clamped symmetrically so that it meets the substrate at a nonzero angle, then the membrane favors an asymmetric shape as water is drawn out through it.
\end{abstract}

DOI: 10.1103/PhysRevE.85.016330

PACS number(s): 47.15.gm, 47.10.A-, 47.20.Ky

\section{INTRODUCTION}

The desiccation and vitrification of biological cells is a proposed method for their long-term preservation [1]. This process has been synthesized for suspensions of individual cells using a technique that involves first loading sugars, such as trehalose, into the cells before removing water from them [2]. Above a critical sugar concentration the contents of the cells undergo vitrification to form a glassy phase in which intracellular processes (and thus the deterioration of the cell) are dramatically slowed. In this paper our aim is to construct a model that describes the dynamic behavior of a cell as it is desiccated, which may provide insight into ways of improving the survival rates of cells when they are subsequently reconstituted (by the reintroduction of water).

Desiccation of an isolated cell may be achieved by surrounding it in a similar-sugar solution of higher concentration than inside the cell. Mammalian cells are bounded by lipid bilayer membranes, which are permeable to small molecules such as water but not to larger molecules such as sugar. Water is drawn out of the cell through osmosis, which causes its volume to decrease. The small bending resistance of the cell's membrane compared to its resistance to longitudinal compression means that the membrane must somehow deform while its surface area is approximately conserved locally [3]. Here we focus on the deformation of the cell's shape as it is desiccated. Another important aspect during desiccation processes is the phase transition of the sugar solution to a glassy phase, and the propagation of the transition front [4,5], but for simplicity we do not consider such effects here.

The first objective is to study the evolution of a cell that is adhered to a substrate in such a way that its contact area is fixed. Recent experiments involving vesicles (closed bilipid membranes), which are artificially fabricated analogs of mammalian cells, have demonstrated that cells may be constrained in this way through chemical patterning of the substrate [6]. Static shapes of these vesicles have also been calculated [6] using an energy minimization technique. Similar methods have been applied to compute static membrane shapes on homogeneous substrates that are flat [7], curved [8], and corrugated [9]. The shapes of isolated vesicles (i.e., away from any boundaries) have also been considered [10]. An alternative formulation, which is analogous to the approach that we take here, is to derive and solve a system of differential equations that describe the static shapes of the membrane [11]. Time-dependent simulations of the motion of free vesicles have been developed using boundary integral [12] and level-set [13] techniques, but to our knowledge these calculations all assume that the volume of the vesicle is conserved and, hence, that there is no transport of water out of the vesicle. In addition, these time-dependent simulations are computationally expensive, and our aim is to develop a simpler model that encapsulates all of the most important dynamical processes that occur.

Membrane deformation has also been considered in several contexts other than the drying of biological cells. These include the onset of wrinkling in an impermeable surfactant monolayer as it is compressed longitudinally [14] and the delamination of a membrane from a substrate caused either by longitudinal compression of the substrate [15] or through osmotic cavitation when a coated substrate is submerged in water [16]. Our model would be applicable to the situation where the liquid beneath the delaminated membrane is subsequently removed through drying.

In this paper we present a simple model that is intended to describe the behavior of a sessile cell as water is drawn out from inside it and to include the physical effects of the membrane and of transport of water across the membrane. This model treats the cell wall as a bilipid membrane that has finite (but small) bending resistance and whose area remains constant. The cell's contents are modeled by a homogeneous solution of sugar and water. Outside the cell is another homogeneous solution of sugar whose concentration is maintained at a constant value that is higher than the initial concentration of sugar inside the cell. Water is drawn out of the cell through the membrane, and we focus on the behavior of the membrane as the volume of the cell decreases. We use a lubrication approximation, and our model is therefore applicable to circumstances in which the membrane height 
is much smaller than its length and where the fluid flow has negligible inertia. Such circumstances occur naturally in the context of the blistering of laminated surfaces when they are submerged, and the behavior of the blisters as they are dried, and we therefore also apply our model to these situations.

The structure of the paper is as follows. In Sec. II we construct the model and derive the governing equations. Whereas our focus is on estimating the behavior of cells during desiccation processes, we also apply our model to understand the behavior of a single blister on an otherwise flat wetting later, and of many blisters that interact through the transport of liquid between them. In Sec. III we begin to analyze these three problems by obtaining equilibrium solutions for the membrane's shape, first in the simpler situation where interactions between the membrane and the substrate are omitted and then in the more realistic situation that there is a short-range wetting potential (repulsive hydration force) between them [17], which prevents the membrane from touching down, and instead leads to the formation of a thin wetting layer between the membrane and the substrate. The variation of these solutions with the external sugar concentration is analyzed, and bifurcations between distinct types of membrane shapes are described. The stability of these solutions to small disturbances is analyzed in Sec. IV, and we describe the dynamically favored configurations of the membrane. We summarize and discuss the results in Sec. V.

\section{PROBLEM DESCRIPTION}

We consider a deformable, semipermeable membrane that lies above a rigid horizontal substrate, as depicted in Fig. 1. In the derivation that follows we use starred variables to denote dimensional quantities and undecorated variables to represent dimensionless quantities. For simplicity we work in a twodimensional geometry, described by Cartesian coordinates $\left(x^{*}, y^{*}\right)$, defined so that the origin lies on the substrate beneath the center of the vesicle. This assumes that there is no variation in the $z^{*}$ direction, so that the membrane's shape would be cylindrical if it were viewed in three dimensions. It would be straightforward to generalize this model to describe an axisymmetric vesicle. From now on it is understood that all quantities that relate directly to the membrane's dimensions (such as volume, area, or water flux through the membrane) are expressed per unit length in the $z^{*}$ direction, so that we use "volume" and "area" to refer, respectively, to the cross-sectional area of the cell and to the length of the membrane measured in the $x^{*}-y^{*}$ plane.

We nondimensionalize vertical length scales with a characteristic vertical length scale $H^{*}$ of the system, such as the initial height of the membrane above the substrate, and horizontal length scales with the horizontal extent $L^{*}$ of the domain. We employ the lubrication approximation [18], which requires $H^{*} \ll L^{*}$, and therefore assume that the dimensionless parameter given by $\epsilon=H^{*} / L^{*}$ is very small. The shape of the membrane is described in dimensionless variables by the surface $y=h(x, t)$, from which it follows that the tangent and normal vectors to this surface are given by

$$
\mathbf{t}=\left(\begin{array}{c}
1 \\
\epsilon h_{x}
\end{array}\right)+O\left(\epsilon^{2}\right) \text { and } \mathbf{n}=\left(\begin{array}{c}
-\epsilon h_{x} \\
1
\end{array}\right)+O\left(\epsilon^{2}\right),
$$

where a subscript indicates differentiation. The dimensionless mean curvature $\mathcal{H}$ is given by

$$
\mathcal{H}=L^{*} \mathcal{H}^{*}=\frac{1}{2} \epsilon h_{x x}+O\left(\epsilon^{3}\right) .
$$

The membrane deforms as water is removed through it, and we denote its local velocity by $\tilde{\mathbf{u}}^{*}\left(x^{*}, t^{*}\right)=\left(\tilde{u}^{*}, \tilde{v}^{*}\right)$. The membrane's resistance to bending is much smaller than its resistance to stretching [3], and so we assume that the membrane's area is locally conserved, so that

$$
\nabla_{s}^{*} \cdot \tilde{\mathbf{u}}_{s}^{*}+\left(\nabla_{s}^{*} \cdot \mathbf{n}\right)\left(\tilde{\mathbf{u}}^{*} \cdot \mathbf{n}\right)=0
$$

where the subscript $s$ denotes projection onto the membrane surface through the operator $\mathbf{I}-\mathbf{n n}$. We nondimensionalize the surface divergence operator with the reciprocal of the horizontal length scale, given by $1 / L^{*}$, and the membrane velocity with a characteristic velocity scale $U^{*}$ that must be determined. We then substitute the above expression for $\mathbf{n}$ to obtain the dimensionless equations [correct to $O\left(\epsilon^{2}\right)$ ]

$$
\begin{aligned}
\mathbf{I}-\mathbf{n n} & =\left(\begin{array}{cc}
1 & \epsilon h_{x} \\
\epsilon h_{x} & 0
\end{array}\right), \\
\nabla_{s} & =\left(\begin{array}{c}
\partial_{x}+h_{x} \partial_{y} \\
\epsilon h_{x} \partial_{x}
\end{array}\right), \quad \text { and } \quad \mathbf{u}_{s}=\left(\begin{array}{c}
\tilde{u}+\epsilon h_{x} \tilde{v} \\
\epsilon h_{x} \tilde{u}
\end{array}\right),
\end{aligned}
$$

[Given a time scale $T^{*}$ of the vesicle's motion, it is intuitive that the dimensional velocity components $\tilde{u}^{*}$ and $\tilde{v}^{*}$ scale, respectively, like $L^{*} / T^{*}$ and $H^{*} / T^{*}$. This implies that the dimensionless velocity component $\tilde{v}$ is an $O(\epsilon)$ factor smaller

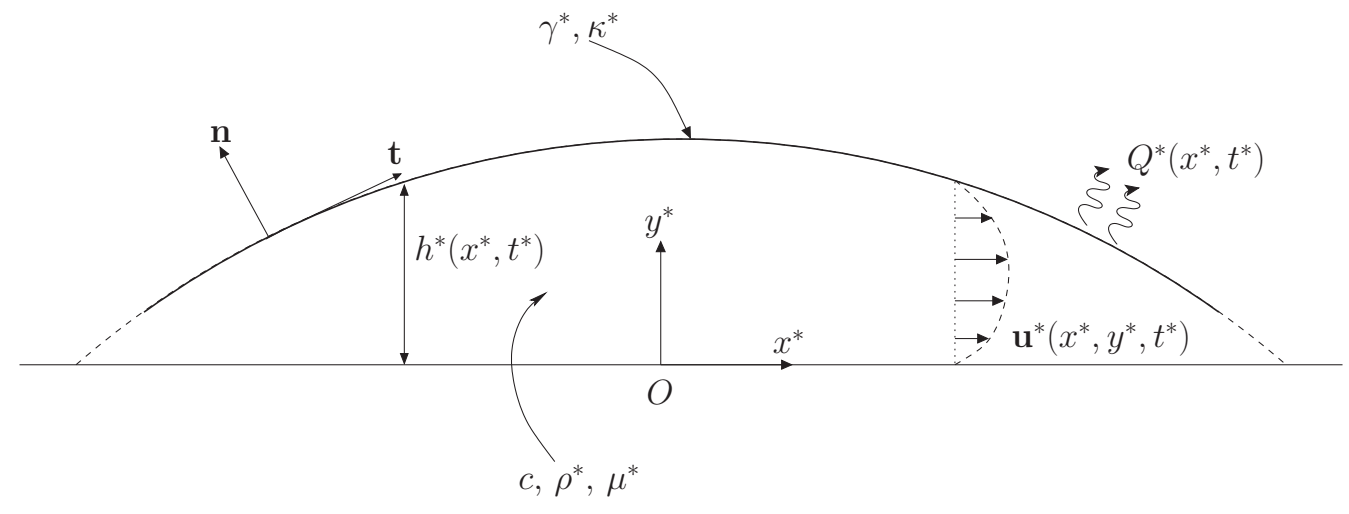

FIG. 1. Diagram of the problem. 
than $\tilde{u}$, and, therefore, that the contribution $\epsilon h_{x} \tilde{v}$ to $\tilde{\mathbf{u}}$ is $O\left(\epsilon^{2}\right)$. From now on, we omit this contribution, and we rescale $\tilde{v}$ so that it is given by $\tilde{v}^{*} T^{*} / H^{*}$ rather than $\tilde{v}^{*} / U^{*}$ and, hence, is $O(\tilde{u})$.] From area conservation (noting that $\tilde{u}_{y}=0$ ) we obtain

$$
\tilde{u}_{x}=O\left(\epsilon^{2}\right)
$$

In addition, the membrane's area must be globally conserved according to

$$
\int_{-1 / 2}^{1 / 2}\left(1+\epsilon^{2} h_{x}^{2}\right)^{1 / 2} d x=\mathcal{L}
$$

where $\mathcal{L}$ is the dimensionless length of the membrane that is not in contact with the substrate. Expansion of this constraint yields

$$
\mathcal{L}=1+\frac{\epsilon^{2}}{2} \int_{-1 / 2}^{1 / 2} h_{x}^{2} d x+O\left(\epsilon^{4}\right)=1+\frac{\epsilon^{2}}{2} \ell+O\left(\epsilon^{4}\right),
$$

where for convenience we have defined the dimensionless "excess membrane length" to be $\ell=\int_{-1 / 2}^{1 / 2} h_{x}^{2} d x$.

The membrane exerts a stress on the liquid surrounding it, which comprises contributions both from its deformation and from the interaction between the membrane and the substrate. This stress must be supported by viscous stress in the liquid, which in dimensional variables is given by

$$
\begin{aligned}
{\left[\boldsymbol{\sigma}^{*} \cdot \mathbf{n}\right]=} & {\left[-\kappa^{*}\left(4 \mathcal{H}^{* 3}+2 \nabla_{s}^{* 2} \mathcal{H}^{*}\right)+2 \gamma^{*} \mathcal{H}^{*}\right] \mathbf{n} } \\
& +\nabla_{s}^{*} \gamma^{*} \mathbf{t}+\boldsymbol{\Pi}^{*}(h) .
\end{aligned}
$$

The parameter $\kappa^{*}$ is the bending stiffness of the membrane, $\gamma^{*}$ is the local tension in the membrane, and $\Pi^{*}$ represents the stress exerted on the liquid owing to the attractive or repulsive interactions between the membrane and the substrate. The first two terms are derived from the Helfrich bending energy [3], under the assumption that the membrane has zero spontaneous curvature (i.e., that it would lie flat in the absence of any external forcing). The third and fourth terms are due to the membrane's tension [19], which varies spatially in order to conserve the membrane's area. (We note that this tension arises as a mechanical response of the membrane to stresses exerted on it, and does not depend directly on the physical properties of the membrane; this is in contrast to the surface tension at liquid-liquid interfaces, which arises owing to the surface energy of the interface independently of any stresses exerted there. Surface tension at the interface between two fluids is typically known, whereas in this problem the tension of the membrane must be determined as part of the problem.) The final term is a short-ranged repulsive term that prevents the membrane from touching down onto the substrate. Such repulsive effects have been observed experimentally $[17,20]$ and - in the absence of electrostatic effects-have been attributed to hydration forces. The precise origin of these forces has been a subject of controversy, but recent molecular dynamics simulations $[21,22]$ support the conclusion that they are caused by the energy cost associated with the disruption of hydrogen-bond networks in water owing to interactions of thin layers of molecules with the membrane and with the substrate. These thin layers have a more pronounced effect as the membrane separation decreases, thereby increasing the repulsive force that results. The hydration force dominates other small-scale membrane-substrate interactions at small distances and for simplicity we, therefore, use the interaction term

$$
\Pi^{*}\left(h^{*}\right)=P_{0}^{*} \exp \left(-h^{*} / \lambda^{*}\right) \mathbf{n},
$$

where $P_{0}^{*}$ represents the amplitude of the (repulsive) hydration stress exerted on the membrane and $\lambda^{*}$ represents the length scale over which it acts. For sufficiently large values of $P_{0}$, the membrane forms a very thin wetting layer rather than touching down. From now on, we use the terms "hydration force" and "wetting potential" interchangeably. We use the leading-order contribution to (3) from bending stress to define a scaling for viscous stress that is given by $\kappa^{*} H^{*} / L^{* 4}$. Then, the viscous stress (3) exerted by the liquid is given at leading order by

$$
\begin{aligned}
{[\boldsymbol{\sigma} \cdot \mathbf{n}]=} & {\left[\gamma h_{x x}-h_{x x x x}+P_{0} \exp (-h / \lambda)\right] \mathbf{n} } \\
& +\epsilon^{-1}\left(\gamma_{x}+h_{x} \gamma_{y}\right) \mathbf{t}+O\left(\epsilon^{2}\right),
\end{aligned}
$$

where

$$
\gamma=\frac{\gamma^{*} L^{* 2}}{\kappa^{*}}
$$

represents the dimensionless tension in the membrane (which must be determined as part of the solution) and

$$
P_{0}=\frac{P_{0}^{*} L^{* 4}}{\kappa^{*} H^{*}} \quad \text { and } \quad \lambda=\frac{\lambda^{*}}{H^{*}},
$$

respectively, represent the dimensionless amplitude and length scale that characterize the variation of hydration stress with the membrane height.

We now consider the flow of liquid beneath the membrane. We treat the liquid beneath the membrane as an aqueous solution of some solute, which is assumed from now on to be sugar. We also assume that the density $\rho^{*}$ and viscosity $\mu^{*}$ of this solution do not change significantly with its concentration.

We estimate the time scale $T^{*}$ of motion by making the usual assumption of lubrication theory [18] that liquid flow is driven primarily by horizontal pressure gradients. The appropriate pressure scale $p_{0}^{*}$ is given by the viscous stress scale $\kappa^{*} H^{*} / L^{* 4}$ obtained above. Horizontal pressure gradients, therefore, scale like $p_{0}^{*} / L^{*}$, and a balance with viscous stress gradients, which scale like $\mu^{*} U^{*} / H^{* 2}$, implies that horizontal velocities scale like $U^{*}=p_{0}^{*} H^{* 2} / \mu^{*} L^{*}$. The time scale of motion is, therefore, given by $T^{*}=U^{*} L^{*}=p_{0}^{*} H^{* 2} / \mu^{*}$, and we nondimensionalize the velocity components $u$ and $v$ of the liquid with the velocity scales

$$
U^{*}=\frac{L^{*}}{T^{*}}=\frac{\kappa^{*} H^{* 3}}{\mu^{*} L^{* 5}} \quad \text { and } \quad V^{*}=\frac{H^{*}}{T^{*}}=\frac{\kappa^{*} H^{* 4}}{\mu^{*} L^{* 6}} .
$$

The dimensionless governing equations for the liquid are then, to $O\left(\epsilon^{2}\right)$,

$$
\begin{aligned}
\operatorname{Re}\left(u_{t}+u u_{x}+v u_{y}\right) & =-p_{x}+u_{y y}, \\
\operatorname{Re} \epsilon^{2}\left(v_{t}+u v_{x}+v v_{y}\right) & =-p_{y},
\end{aligned}
$$

where

$$
\operatorname{Re}=\frac{\rho^{*} U^{*} H^{*}}{\mu^{*}}\left(\frac{H^{*}}{L^{*}}\right)
$$

is a reduced Reynolds number. In the lubrication approximation, it is usual to omit the inertial terms on the left-hand sides 
of these equations, which is valid provided $\mathrm{Re} \ll 1$ (which we demonstrate in Appendix A). The simplified equations are then given by

$$
\begin{gathered}
p_{x}=u_{y y}, \\
p_{y}=0, \\
u_{x}+v_{y}=0,
\end{gathered}
$$

where (10) enforces the incompressibility of the liquid. On the substrate there is no slip and no penetration, so that

$$
u=v=0 \quad \text { at } \quad y=0 .
$$

Beneath the membrane, the viscous-stress tensor is given in dimensionless variables by

$$
\sigma=\left(\begin{array}{c}
-p \epsilon u_{y} \\
\epsilon u_{y}-p
\end{array}\right)+O\left(\epsilon^{2}\right) .
$$

The viscous stress components of the liquid outside the membrane are much smaller than those inside when $\epsilon \ll 1$. To see this, consider an otherwise quiescent flow outside the membrane driven by the growth of undulations in the membrane's shape such that the undulations have horizontal length scale $L^{*}$ and move with a velocity scale that is given by $V_{m}^{*}=H^{*} / T^{*}$. It can be shown that the velocity components scale like $V_{m}^{*}$ near the membrane but decay away from the membrane with characteristic vertical length scale $L^{*}$. It follows that the viscous stress in the liquid outside the membrane scales like $\mu V_{m}^{*} / L^{*} \sim \mu H^{*} U^{*} / L^{* 2}$. The corresponding component of viscous stress beneath the membrane scales like $\mu U^{*} / H^{*}$ and is therefore an $O\left(\epsilon^{2}\right)$ factor larger. (A more rigorous estimate of the size of the viscous stress above the membrane may be obtained by adapting the analysis of, for example, [23], which yields identical results.) We, therefore, neglect the dynamic effects of the fluid above the membrane in the analysis that follows.

The relevant viscous-stress tensor components at the membrane are, to $O\left(\epsilon^{2}\right)$,

$$
\begin{array}{r}
\mathbf{n} \cdot \boldsymbol{\sigma} \cdot \mathbf{n}=-p, \\
\mathbf{t} \cdot \boldsymbol{\sigma} \cdot \mathbf{n}=\epsilon u_{y} .
\end{array}
$$

By comparing these expressions with the viscous stress (5), it follows that

$$
\begin{gathered}
p=h_{x x x x}-\gamma h_{x x}-P_{0} \exp (-h / \lambda) \text { at } y=h, \\
u_{y}=\epsilon^{-2}\left(\partial_{x}+h_{x} \partial_{y}\right) \gamma \quad \text { at } y=h .
\end{gathered}
$$

The tangential stress condition (13) implies that there is no tension variation in the membrane at leading order and, hence, that the tension is given by

$$
\gamma=\gamma_{0}(t)+\epsilon^{2} \gamma_{1}[x, h(x, t), t]+o\left(\epsilon^{2}\right)
$$

We obtain the velocity profile of the liquid by first integrating the velocity equation (8) subject to the no-slip condition (11a) and the tangential-stress condition (13). This yields

$$
u(x, y, t)=\frac{1}{2}\left[\left(y^{2}-2 h y\right) p_{x}+2 y \gamma_{1, x}\right],
$$

where the pressure $p$ is independent of $y$ from (9) and, hence, given by (12). We substitute this expression into the incompressibility condition (10) and integrate subject to the no-penetration condition (11b) to obtain

$$
v(x, y, t)=-\frac{y^{3}}{6} p_{x x}+\frac{y^{2}}{2}\left(h p_{x}-\gamma_{1, x}\right)_{x} .
$$

We now consider the motion of the membrane. The fluid flow must satisfy the no-slip condition on the membrane so that the tangential membrane velocity satisfies

$$
\left(\left.\mathbf{u}\right|_{y=h}-\tilde{\mathbf{u}}\right) \cdot \mathbf{t}=0 .
$$

The permeability of the membrane to water means that liquid passes through it according to

$$
\left(\left.\mathbf{u}\right|_{y=h}-\tilde{\mathbf{u}}\right) \cdot \mathbf{n}=Q(x),
$$

where $Q(x)$ is the volume flux of water out of the membrane and is driven by pressure and concentration gradients across the membrane. Expansion of these boundary conditions in the limit $\epsilon \ll 1$ yields

$$
u=\tilde{u}+O\left(\epsilon^{2}\right) \quad \text { and } \quad v=\tilde{v}+Q+O\left(\epsilon^{2}\right) \quad \text { at } \quad y=h .
$$

From (15) it then follows that the membrane's horizontal velocity is given by $\tilde{u}=h \gamma_{1, x}-\frac{1}{2} h^{2} p_{x}$, and substitution into the area conservation constraint (1) yields

$$
\left(h \gamma_{1, x}-\frac{h^{2}}{2} p_{x}\right)_{x}=0
$$

which describes how the tension varies spatially in order to ensure that the membrane is not stretched by viscous stresses exerted on it. Hence, the spatial variation of tension in Eq. (14) may be eliminated in favor of $p_{x}$ so that only the mean tension $\gamma_{0}$ remains unknown.

The evolution of the membrane's shape is given by the kinematic relation $h_{t}=\tilde{v}-\tilde{u} h_{x}$, and from (16) and (15) this implies that, at leading order,

$$
\begin{aligned}
h_{t} & =\left.v\right|_{y=h}-\left.u\right|_{y=h} h_{x}-Q=-\left(\int_{0}^{h} u d y\right)_{x}-Q \\
& =\left(-\frac{h^{3}}{3} p_{x}+\frac{h^{2}}{2} \gamma_{1, x}\right)_{x}-Q=\left(\frac{h^{3}}{12} p_{x}\right)_{x}-Q,
\end{aligned}
$$

where the final equality is obtained through substitution for the tension variation using (17). We note that this result is identical to the standard lubrication result that would have been obtained were the no-slip condition $u=0$ prescribed both on the membrane and on the substrate.

Finally, we express the composition of the solution beneath the membrane as a volume fraction $c_{\text {in }}$, so that $c_{\text {in }}=0$ corresponds to there being only water present, whereas $c_{\text {in }}=1$ represents there being no water present. As described in Appendix A, the liquid flow beneath the membrane has very small Peclet number, so that diffusive transport takes place much faster than advective transport and $c_{\text {in }}$ may be treated as spatially constant. However, $c_{\text {in }}$ does vary in time owing to the transport of water through the membrane. Although water is lost, the impermeability of the membrane to the sugar means that the total amount of sugar beneath the membrane remains 
conserved. Hence,

$$
c_{\text {in }} \int_{-1 / 2}^{1 / 2} h d x=\bar{S}
$$

where $\bar{S}$ is a dimensionless constant. As described in Appendix A, the transport of water across the membrane is due both to hydrostatic pressure gradients and to osmotic pressure gradients. The total of these contributions is given (in dimensional variables) by

$$
Q^{*}(x)=k_{1}^{*}\left(c_{\text {out }}-c_{\text {in }}\right)+k_{2}^{*}\left(p_{\text {in }}^{*}-p_{\text {out }}^{*}\right) .
$$

The terms $p_{\text {in }}^{*}$ and $p_{\text {out }}^{*}$ represent the pressure on either side of the membrane. Because we have omitted the dynamic effects of the liquid outside the membrane, we may set $p_{\text {out }}^{*}=0$ and, thus, identify the dimensionless pressure difference $p_{\text {in }}-p_{\text {out }}$ with the pressure $p$ given by (12). We nondimensionalize the flux $Q^{*}$ per unit area using the vertical velocity scale $V^{*}$, so that in dimensionless variables

$$
Q(x)=k_{1}\left(c_{\text {out }}-c_{\text {in }}\right)+k_{2} p,
$$

where the dimensionless parameters $k_{1}$ and $k_{2}$ are positive and are given by

$$
k_{1}=\frac{k_{1}^{*}}{V^{*}}=\frac{k_{1}^{*} \mu^{*} L^{* 6}}{\kappa^{*} H^{* 4}} \quad \text { and } \quad k_{2}=\frac{k_{2}^{*} p_{0}^{*}}{V^{*}}=\frac{k_{2}^{*} \mu^{*} L^{* 2}}{H^{* 3}} .
$$

\section{A. Summary of governing equations and dimensionless parameters}

Here we collect together the equations that govern the evolution of the membrane's shape and the water concentration beneath the membrane. From (18), (21), and (12) the height evolution equation is given by

$$
h_{t}=\left(\frac{h^{3}}{12} p_{x}\right)_{x}-k_{1}\left(c_{\text {out }}-c_{\text {in }}\right)-k_{2} p,
$$

where the pressure $p$ is given at leading order by

$$
p=h_{x x x x}+\Gamma h_{x x}-P_{0} \exp (-h / \lambda),
$$

and where we have denoted, for convenience, the membrane's compression using $\Gamma=-\gamma_{0}$.

In the following sections we consider three prototypical problems which are depicted in Fig. 2 and which we describe in greater detail in Sec. II B. For each of these problems, the sugar concentration $c_{\text {out }}$ outside the membrane is assumed to be fixed, and the sugar concentration $c_{\text {in }}$ and the membrane compression $\Gamma$ vary in order to satisfy the integral constraints
(2) and (19), which depend on the values of $\ell$ and $\bar{S}$. For each problem, we take $\ell=16 / 3$ and $\bar{S}=2 c_{\text {init }} / 3 \approx 6.7 \times 10^{-3}$, where $c_{\text {init }}=10^{-2}$ is the initial sugar concentration underneath the membrane. The integral constraints (2) and (19) are, therefore, given by

$$
\int_{-1 / 2}^{1 / 2} h_{x}^{2} d x=\frac{16}{3} \text { and } \int_{-1 / 2}^{1 / 2} h d x=\frac{2 c_{\text {init }}}{3 c_{\text {in }}} .
$$

These values are chosen for consistency with the parabolic-cap shape that is relevant when $c_{\text {init }}>c_{\text {out }}$, and which is analogous to the spherical-cap shape taken by three-dimensional vesicles of moderate aspect ratio [7]. The dimensions of the vesicle have been nondimensionalized so that the vesicle initially has unit height and unit length, and it follows that the appropriate parabolic-cap solution is given by

$$
h=1-4 x^{2} \text { and } c_{\text {in }}=c_{\text {init }} .
$$

In Appendix A, we describe the parameter values that are representative of experiments in which biological cells are dried. The parameter values that we use in later sections are chosen for ease of computation and presentation of results; although they are in some cases significantly different to those obtained in Appendix A, we expect that they should give a good qualitative description of the evolution of the vesicle. In particular, the value of $k_{2}$ that we use is much larger than those typical of vesicle drying experiments. If $k_{2} \approx 0$, then (23a) implies that the volume loss of the vesicle is driven almost entirely by the concentration difference $c_{\text {in }}-c_{\text {out }}$ across the membrane, whereas hydrostatic pressure inside the vesicle plays a negligible role. Because $k_{1}$ is very large, the vesicle's volume decreases (and $c_{\text {in }}$ approaches $c_{\text {out }}$ ) on a very short time scale while the membrane wrinkles owing to its incompressibility. As we describe in Sec. IV A 1, many of the wrinkling modes are unstable when $c_{\text {out }}$ is much larger than $c_{\text {in }}$, and the temporal variation of the membrane's shape is, therefore, very sensitive to disturbances. We, instead, focus on the behavior of the vesicle toward the end of the desiccation process when $k_{1}\left(c_{\text {in }}-c_{\text {out }}\right) \sim k_{2} p$, so that the concentration-driven and pressure-driven terms in Eq. (23a) are comparable. Although the membrane is likely to become very wrinkled while $c_{\text {in }}$ rapidly approaches $c_{\text {out }}$, we show in Sec. IV A 3 that these wrinkles subsequently coarsen rapidly in order to reduce the membrane's compression. We, therefore, focus on membrane shapes that have few wrinkles. Our use of a relatively large value of $k_{2}$ is for ease of presentation, as it yields predictions of $c_{\text {in }}$ whose differences from $c_{\text {out }}$ are much larger (and, hence, more easily distinguishable) than would be (a)

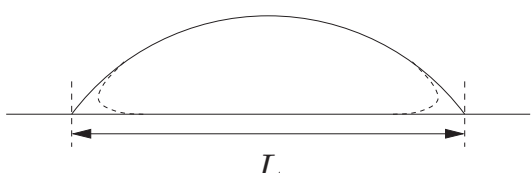

$L$ (b)

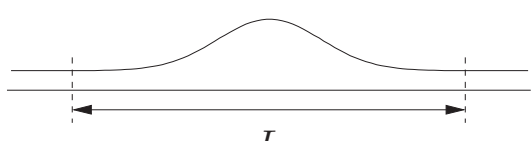

$L$

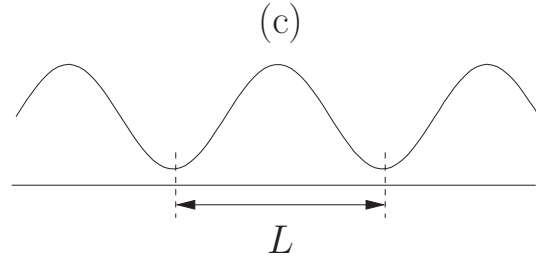

FIG. 2. Schematic diagrams of the three problems considered in this paper. (a) Problem I: a vesicle that is adhered to a substrate. (b) Problem II: an isolated blister in a membrane that coats a substrate. (c) Problem III: the buckling of a long film that lies on a cushion of liquid. 
observed experimentally. We expect that out analysis applies when $c_{\text {in }}$ is very close to $c_{\text {out }}$ and that, despite the difference in the quantitative value of $k_{2}$ used, the conclusions drawn in the following sections are qualitatively accurate.

\section{B. Boundary conditions}

Equations (23a) and (23b) comprise a sixth-order system of differential equations with two unknown parameters, $c_{\text {in }}$ and $\Gamma$, which are determined by the constraints $(23 \mathrm{c}, \mathrm{d})$. Six boundary conditions are therefore needed at the edges of the membrane to complete each problem.

\section{Problem I: The drying of a vesicle that is adhered to a substrate}

The first problem is intended to represent a vesicle that is attached to a rigid flat substrate, as depicted in Fig. 2(a). We note that the lubrication approximation breaks down near the extremities of the vesicle because the membrane must turn sharply there in order to lie flat on the substrate [7], as shown by the dashed lines. A rigorous derivation of appropriate boundary conditions that encapsulate the membrane's shape in these regions is currently under way, and will be reported in a later publication. Here we instead prescribe "clamped" conditions that fix the angle and the height of the membrane at the edges and assume that these conditions remain valid throughout the drying process. The angle prescribed is derived using the parabolic-cap shape given by (24a), and we prescribe the conditions

$$
h=0 \quad \text { and } \quad h_{x}=\mp 4 \quad \text { at } \quad x= \pm \frac{1}{2} .
$$

These boundary conditions imply that $h=4\left(x \pm \frac{1}{2}\right)+$ $O\left(x^{2}\right)$ and that $h_{t}=O\left(x^{2}\right)$ as $x \rightarrow \mp \frac{1}{2}$. Substitution of these expressions into (23a) reveals that there are essential singularities at $x= \pm \frac{1}{2}$, and application of the method of dominant balance shows that (23a) admits a solution for which the pressure diverges like $\exp \left[\sqrt{3 / 4\left(x \mp \frac{1}{2}\right)}\right]$ near the edges. This pressure cannot be balanced by stresses in the membrane; the divergent solution is therefore unphysical and must be suppressed by prescribing the boundary condition

$$
p_{x}=0 \quad \text { at } \quad x= \pm \frac{1}{2} \text {. }
$$

\section{Problem II: The drying of an isolated blister in a membrane}

The second problem represents the removal of water from beneath an isolated blister in a membrane that is otherwise flat, as depicted in Fig. 2(b). Such blisters have been observed experimentally in gel-coated laminates when they are submerged in water [16]. The analysis here would be applicable to the evolution of this blister if it were subsequently dried out.

We assume that away from the blister there is a thin, flat, wetting layer that is at rest. The height of this layer is governed by a balance between the repulsive hydration stress and the attractive osmotic pressure. We, therefore, prescribe the conditions

$$
\begin{gathered}
P_{0} \exp (-h / \lambda)=\frac{k_{1}}{k_{2}}\left(c_{\text {out }}-c_{\text {in }}\right), \\
h_{x}=0 \text { and } \quad p_{x}=0 \text { at } x= \pm \frac{1}{2} .
\end{gathered}
$$

\section{Problem III: The formation of multiple blisters in a long membrane}

The final problem is intended to represent the situation in which a membrane lies on a thin carrier layer of liquid above a substrate and forms multiple blisters, as depicted in Fig. 2(c). The onset of wrinkle formation as a long membrane is compressed has previously been analyzed in the context of the transfer of membranes to substrates on a carrier layer of liquid [14]. Here we intend to describe the evolution of such membranes in the case that water is then removed through the membrane.

For this problem we prescribe symmetry conditions on the edges of the domain, which are given by

$$
h_{x}=h_{x x x}=p_{x}=0 \quad \text { at } \quad x= \pm \frac{1}{2} .
$$

\section{EQUILIBRIUM MEMBRANE SHAPES}

We first use our model to predict equilibrium membrane shapes and their dependence on the external sugar concentration $c_{\text {out }}$. Two necessary conditions for equilibrium are that there be zero pressure gradients (which would otherwise drive fluid flow beneath the membrane) and that there be zero net volume flux through the membrane, so that

$$
p_{x} \equiv 0 \quad \text { and } \quad k_{2} p=-k_{1}\left(c_{\mathrm{out}}-c_{\text {in }}\right) .
$$

Together with (23b), these imply that equilibrium membrane shapes must satisfy

$$
h_{x x x x}+\Gamma h_{x x}-P_{0} \exp (-h / \lambda)=-\frac{k_{1}}{k_{2}}\left(c_{\text {out }}-c_{\text {in }}\right),
$$

subject to the integral constraints $(23 \mathrm{c}, \mathrm{d})$ and the constraints given by either (25), (26) or (27), according to which of the three problems is under consideration.

For each problem we vary the external water concentration $c_{\text {out }}$ while keeping the parameters $P_{0}, \lambda, k_{1}$, and $k_{2}$ fixed. The membrane compression $\Gamma$ and the sugar concentration $c_{\text {in }}$ inside the membrane are determined as part of the solution. We calculate numerical solutions to this equation using AUTO07P, a software package which implements a continuation (homotopy) method [24]. Such a method begins with an initial solution that must typically be found analytically. In the following section we describe how, in the absence of hydration stresses, it is possible to obtain exact solutions to (28) through solution of a system of algebraic equations that represent the boundary conditions and integral constraints. These solutions primarily serve as initial solutions to which the hydration stress may then be gradually reintroduced.

\section{A. Solutions in the absence of hydration stresses}

In the absence of hydration stresses, and when $\Gamma>0$, the shape equation (28) for the membrane has general solution given by

$$
\begin{aligned}
h= & \frac{k_{1}\left(c_{\text {out }}-c_{\text {in }}\right)}{8 k_{2} \Gamma}\left(1-4 x^{2}\right)+A \cos (\sqrt{\Gamma} x)-\alpha \\
& +B \sin (\sqrt{\Gamma} x)-\beta x .
\end{aligned}
$$

The constants $A$ and $B$ represent amplitudes of the membrane's bending modes, and $\alpha$ and $\beta$ are other integration constants. 
Together with $c_{\text {in }}$ and $\Gamma$, these constants are determined by the boundary conditions and integral constraints. We note that the wavelength of the bending mode is related to the membrane's compression according to $L_{B} \propto \Gamma^{-1 / 2}$. This reflects the intuitive result that as the membrane's compression increases, the membrane becomes more wrinkled so that the larger compression may be supported by bending stress.

The membrane's shape may be thought of as comprising a symmetric contribution (both from the parabolic-cap shape and from the terms premultiplied by $A$ and $\alpha$ ) and an antisymmetric contribution (from the terms premultiplied by $B$ and $\beta$ ). It is convenient to treat these two types of contributions separately when considering the boundary conditions prescribed at the edges of the membrane.

We note that without wetting interactions (i.e., $P_{0}=0$ ), some of these solutions have regions where $h<0$, so that the membrane has "passed through" the substrate. We, nonetheless, discuss these solutions briefly in order to provide contrast with the physical case where $0<\lambda \ll 1$ and $P_{0} \gg 1$.

Substitution of the solution (29) for the membrane's shape into the constraints $(23 \mathrm{c}, \mathrm{d})$ for the membrane's area and for the amount of sugar beneath the membrane, respectively, yields

$$
\begin{aligned}
& \frac{k_{1}^{2}\left(c_{\text {out }}-c_{\text {in }}\right)^{2}}{12 k_{2}^{2} \Gamma^{2}}+A^{2} \Gamma\left(\frac{1}{2}-\frac{1}{2 \sqrt{\Gamma}} \sin \sqrt{\Gamma}\right) \\
& +\frac{2 A k_{1}\left(c_{\text {out }}-c_{\text {in }}\right)}{k_{2} \sqrt{\Gamma}}\left(\frac{2}{\Gamma} \sin \frac{\sqrt{\Gamma}}{2}-\frac{1}{\sqrt{\Gamma}} \cos \frac{\sqrt{\Gamma}}{2}\right) \\
& +B^{2} \Gamma\left(\frac{1}{2}+\frac{1}{2 \sqrt{\Gamma}} \sin \sqrt{\Gamma}\right)+\beta^{2}-4 B \beta \sin \frac{\sqrt{\Gamma}}{2}=\frac{16}{3},
\end{aligned}
$$

and

$$
\frac{k_{1}\left(c_{\mathrm{out}}-c_{\mathrm{in}}\right)}{12 k_{2} \Gamma}+\frac{2 A}{\sqrt{\Gamma}} \sin \left(\frac{\sqrt{\Gamma}}{2}\right)-\alpha=\frac{2 c_{\mathrm{init}}}{3 c_{\mathrm{in}}} .
$$

Each of the three problems requires that the membrane's slope at the edges be given by $h_{x}=\mp M$ at $x= \pm \frac{1}{2}$, where $M=4$ for Problem I and $M=0$ for Problems II and III. This yields

$$
\begin{aligned}
\frac{k_{1}\left(c_{\mathrm{out}}-c_{\mathrm{in}}\right)}{2 k_{2} \Gamma}+A \sqrt{\Gamma} \sin \left(\frac{\sqrt{\Gamma}}{2}\right) & =M, \\
B \sqrt{\Gamma} \cos \left(\frac{\sqrt{\Gamma}}{2}\right)-\beta & =0 .
\end{aligned}
$$

Problems I and II have conditions prescribed on the membrane's height at the edges. For Problem II, if $P_{0}$ is sufficiently large then the hydration stress ensures that the membrane lies above the substrate. If we were to simply set $P_{0}=0$, then the boundary condition (26a) would imply that equilibrium solutions exist only when $c_{\text {in }}=c_{\text {out }}$, as it would otherwise be impossible to balance the osmotic pressure away from the blister. To obtain solutions that more closely resemble those for which hydration stresses are present, we replace this condition by instead prescribing that $h=0$ at $x= \pm \frac{1}{2}$. This condition is identical to that prescribed for Problem I, and it follows that solutions to both problems must satisfy the constraints

$$
\alpha=A \cos \left(\frac{\sqrt{\Gamma}}{2}\right) \text { and } \beta=2 B \sin \left(\frac{\sqrt{\Gamma}}{2}\right) .
$$

Problem III, instead, has the condition that $h_{x x x}=0$ at $x= \pm \frac{1}{2}$. The corresponding solutions must therefore satisfy the constraints

$$
A \sin \left(\frac{\sqrt{\Gamma}}{2}\right)=B \cos \left(\frac{\sqrt{\Gamma}}{2}\right)=0 .
$$

In the absence of hydration stresses between the membrane and the substrate, the six constraints [given by (30)-(32) together with (33) for Problems I and II and (34) for Problem III] fully determine the membrane's shape via (29). We obtain solutions for problems I and II using AUTO-07P. As a preliminary step, we eliminate $\alpha$ and $\beta$ in Eqs. (30)-(32) using (33) to simplify the system to four equations for the unknown variables that remain. We then use the initial solution given by a parabolic cap, whose shape and parameter values are given by

$$
\begin{aligned}
h & =1-4 x^{2}, \quad A=B=0, \quad c_{\text {in }}=c_{\text {init }}, \quad \text { and } \\
\Gamma & =\frac{k_{1}\left(c_{\text {out }}-c_{\text {init }}\right)}{8 k_{2}} .
\end{aligned}
$$

It is straightforward to show that these parameter values satisfy all four constraints (30)-(32) when $M=4$ in the slope constraint (32a). This solution is, therefore, valid for Problem I. For Problem II, a valid solution may be obtained by first adjusting the value of $M$ to zero. Once an appropriate initial solution has been obtained, the external sugar concentration $c_{\text {out }}$ is gradually adjusted while the values of $A, B, c_{\text {in }}$, and $\Gamma$ are allowed to vary freely. Once these values are determined, the solution (29) is used as an initial solution to the differential equation (28) with $P_{0}=0$, for values of $c_{\text {out }}$ that are small enough that $h \gtrsim-\lambda$ everywhere. The value of $P_{0}$ may then be adjusted to the desired value. [The restriction on $c_{\text {out }}$ is necessary since the step size required by the continuation method is prohibitively small owing to the sensitivity of (28) to $P_{0}$ when $h \lesssim-\lambda$.]

It is much simpler to derive solutions to Problem III than for Problems I and II in the case where $P_{0}=0$. In this case, equilibrium solutions exist only when $c_{\text {in }}=c_{\text {out }}$, because otherwise the quadratic term in Eq. (29) would violate the symmetry condition (27b) prescribed at $x= \pm \frac{1}{2}$. Substitution of (29) and $c_{\text {in }}=c_{\text {out }}$ into (27) then implies that

$$
A \sin \left(\frac{\sqrt{\Gamma}}{2}\right)=B \cos \left(\frac{\sqrt{\Gamma}}{2}\right)=\beta=0 .
$$

Because $c_{\text {in }}=c_{\text {out }}$, the area constraint (30) implies that either $A \neq 0$ or $B \neq 0$, and hence that

$$
\Gamma_{n}=n^{2} \pi^{2} \quad \text { and } \quad\{A, B\}= \begin{cases}\left\{0, \sqrt{\frac{32}{3 \Gamma_{n}}}\right\}, & n \text { odd }, \\ \left\{\sqrt{\frac{32}{3 \Gamma_{n}}}, 0\right\}, & n \text { even }\end{cases}
$$

where $n$ is a positive integer that parametrizes the different families of solutions. We refer to successive branches using roman numerals in the following sections. 
Finally, the volume constraint (31) implies that $\alpha$ is given by

$$
\alpha=\frac{2 c_{\text {init }}}{3 c_{\text {in }}} .
$$

If $\alpha$ were less than $A$ or $B$, then the membrane would pass beneath $h=0$, making it difficult to directly compute solutions for nonzero values of $P_{0}$ owing to the high sensitivity of the wetting potential on $P_{0}$ when $h<0$. We, therefore, instead begin with a solution that has a smaller value of $\ell$. This has the effect of replacing the value of $A$ or $B$ by the smaller value $\sqrt{2 \ell / \Gamma_{n}}$, where $\ell$ may be selected so that $\alpha>\max \{A, B\}$. The hydration stress is then reintroduced by setting $P_{0}$ to the desired value and then adjusting the value of $\ell$ back to $16 / 3$ so that the area constraint (23c) is again satisfied.

\section{B. Results}

We now describe the equilibrium solutions for each of the three problems in turn, in either case that the hydration stresses between the membrane and the substrate is absent or present.

\section{Problem I}

Figure 3(a) shows how the sugar concentration $c_{\text {in }}$ varies along each solution branch as $c_{\text {out }}$ is increased for Problem I in the case where $P_{0}=0$, and Fig. 3(b) shows particular membrane shapes. When $c_{\text {out }} \approx c_{\text {init }}$ there is a unique solution for which the membrane has a parabolic-cap shape and the bending amplitudes $A$ and $B$ are both zero. This branch persists as $c_{\text {out }}$ is increased. All solutions on this branch have $c_{\text {in }}=c_{\text {init }}$ (where $c_{\text {init }}=0.01$ in Fig. 3) owing to the volume constraint (23d). It follows that the osmotic pressure drop across the membrane increases as $c_{\text {out }}$ is increased. This pressure drop is balanced by its compression $\Gamma$, and both quantities increase with $c_{\text {out }}$. There are several bifurcation points, shown in Fig. 3(a), which occur when $\Gamma$ attains one of several values (described below) that permit nontrivial bending modes.
The first bifurcation encountered is subcritical and leads to a branch that represents asymmetric solutions. Substitution of the constraint (33b) for the membrane's height into the constraint (32b) for the membrane's slope yields

$$
B\left[\frac{\sqrt{\Gamma}}{2} \cos \left(\frac{\sqrt{\Gamma}}{2}\right)-\sin \left(\frac{\sqrt{\Gamma}}{2}\right)\right]=0 .
$$

The bifurcation point occurs when $B=0$ and $\Gamma \approx 81$, for which value the square-bracketed expression is zero. One of the branches represents parabolic-cap solutions, for which $\Gamma \gtrsim 81$ and $B$ remains zero, whereas the second branch represents asymmetric solutions for which the bending amplitude $B$ is nonzero but $\Gamma$ remains constant. Along the second branch, because $\Gamma$ is fixed the amplitude $A$ of the symmetric bending mode and the internal sugar concentration $c_{\text {in }}$ are determined together by the volume constraint (31) and the constraint (32a) on the slope of the membrane at either edge. Finally, the amplitude $B$ of the asymmetric bending mode is determined by the area constraint (30). There are similar subcritical bifurcations from parabolic-cap solutions toward asymmetric solutions, each of which occur at the other (discrete) values of $\Gamma$ for which the square-bracketed expression in Eq. (35) is zero.

The asymmetric solution branches terminate when they meet another branch, which represents a third type of solution. We discuss these "dimpled" solutions in greater detail below. Figure 3(bII,III) shows how the asymmetry of the membrane shapes initially grows as the branch is traversed away from the parabolic-cap solution branch before the membrane's shape subsequently approaches a symmetric dimpled shape.

If the parabolic-cap branch were traversed by increasing $c_{\text {out }}$ past the first bifurcation point described above, it would eventually encounter a second bifurcation toward a branch that represents symmetric dimpled solutions. For these solutions, the amplitude $B$ of the asymmetric bending mode is zero, whereas the amplitude $A$ of the symmetric mode is nonzero. We follow a similar process as for our analysis of the asymmetric branch and combine the constraints (32a) and (33a) for the height and slope of the membrane with the volume
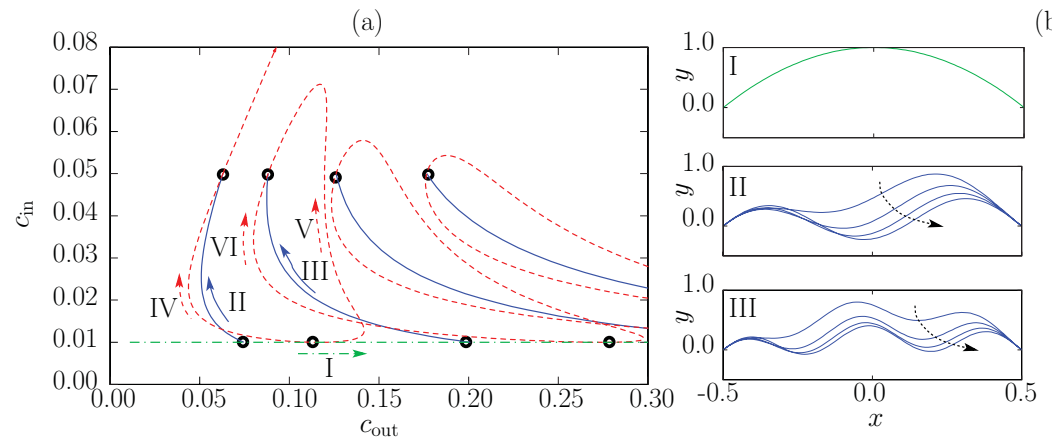

(b)
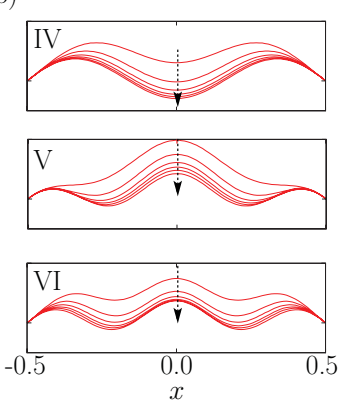

FIG. 3. (Color online) Equilibrium solutions for Problem I with $P_{0}=0$. The other parameter values used are $\ell=16 / 3, \bar{S}=2 / 300$ (so that $c_{\text {init }}=10^{-2}$ ), $k_{1}=10^{2}$, and $k_{2}=10^{-2}$. (a) The variation of internal sugar concentration $c_{\text {in }}$ as the external sugar concentration $c_{\text {out }}$ is varied. Circles denote bifurcation points, and arrows denote the variation of the membrane shapes [shown in (b)] as the branch is traversed (see text). The chain-dashed branch (labeled I) represents the parabolic-cap solution, the solid branches (two of which are labeled II and III) represent asymmetric solutions, and the dashed branch (three portions of which are labeled IV-VI) represents symmetric solutions. (b) Equilibrium membrane shapes. Arrows show the direction in which the branches are traversed in (a), and solutions are plotted for $c_{\text {in }}=0.015$ and multiples of 0.01 thereafter. 
constraint (31) to obtain

$$
\begin{aligned}
A & {\left[\left(\frac{2}{\sqrt{\Gamma}}-\frac{\sqrt{\Gamma}}{6}\right) \sin \left(\frac{\sqrt{\Gamma}}{2}\right)-\cos \left(\frac{\sqrt{\Gamma}}{2}\right)\right] } \\
& =\frac{2 c_{\text {init }}-2 c_{\text {in }}}{3 c_{\text {in }}} .
\end{aligned}
$$

Along the parabolic-cap branch, $A=0$ and $c_{\text {in }}=c_{\text {init }}$. The bifurcations toward the dimpled solution branch occur when the membrane's compression $\Gamma$ takes a value for which the square-bracketed expression is zero. In a similar way to the bifurcations toward asymmetric solutions, one branch corresponds to the parabolic-cap solutions, has $A=0$, with the membrane's compression $\Gamma$ varying freely, and there is a subcritical bifurcation toward a branch that represents dimpled solutions with $A \neq 0$. However, there is an additional supercritical bifurcation toward dimpled solutions. Along the first subcritical branch, labeled IV in Fig. 3, the membrane's compression decreases [so that the square-bracketed expression in Eq. (36) is negative] while the bending amplitude $A$ is negative and increases in magnitude. Conversely, along the first supercritical branch (labeled V in Fig. 3) the membrane's compression increases while $A$ is positive and increases. As for the bifurcations toward asymmetric solutions, there are several bifurcations from the parabolic-cap branch toward dimpled solutions. Each supercritical branch smoothly meets the subcritical branch that originates from the next-highest bifurcation point, and the dimpled solutions may be thought of as a single continuous family of solutions.

We note that the solution branches shown in Fig. 3(a) seem to cross at several points other than the primary bifurcation points labeled. These are not crossing points but are, instead, a result of the projection of the solutions onto the $\left(c_{\mathrm{out}}, c_{\mathrm{in}}\right)$ plane.

Figure 4 shows how the equilibrium solutions are modified by the presence of hydration stresses. When $c_{\text {out }} \approx c_{\text {init }}$, hydration stress is important only in very thin regions near the edges and the solutions obtained are similar to those shown in Fig. 3 for the case $P_{0}=0$. As for the case where hydration stress is absent, the first bifurcation is toward a branch that represents asymmetric solutions. Along this branch, the membrane's shape and sugar concentration $c_{\text {in }}$ are initially very close to those in the case $P_{0}=0$. However, for values of $c_{\text {out }}$ for which the membrane approaches the substrate, hydration stresses impede the further removal of water from the vesicle. Figure 3 demonstrates how, above a critical value of $c_{\text {in }}$, further increase of $c_{\text {in }}$ takes place very slowly as $c_{\text {out }}$ is increased. The critical value corresponds to the situation where the minimum height (away from the edges) is such that $P_{0} \exp \left(-h_{\min } / \lambda\right)$ is comparable to $k_{1}\left(c_{\text {in }}-c_{\text {out }}\right) / k_{2}$ in Eq. (28). This conclusion is supported additional calculations, not shown here, which suggest that $h_{\min }$ increases both with $P_{0}$ and with $\lambda$ (holding all other parameter values fixed) and also that the critical value of $c_{\text {in }}$ decreases as $P_{0}$ and $\lambda$ is increased. Above this critical value, the main effect of increasing $c_{\text {out }}$ is no longer to simply increase $c_{\text {in }}$, but rather to press the membrane against the substrate, which increases the membrane's compression and slightly decreases $h_{\text {min }}$. The increased compression of the membrane supports a larger osmotic pressure drop across it, and, therefore, a smaller value of $c_{\text {in }}$.

In contrast to the case where there is no hydration stress, the first asymmetric branch does not terminate but instead persists for arbitrarily large values of $c_{\text {out }}$. As shown in Fig. 4(bII), solutions on this branch form two lobes, of different sizes, which are separated by a wetting layer. As $c_{\text {out }}$ is increased, water is drawn out of both lobes and so their sizes do not equalize. In contrast, the other asymmetric branches do terminate, as exemplified in Fig. 4(bIII). As $c_{\text {out }}$ is increased, one dimple is first pressed against the substrate before the corresponding dimple on the other side is pressed down to match. These solutions therefore reattain symmetry and the solution branches again terminate onto the dimpled solution branch.

In the presence of hydration forces there are no longer bifurcations between parabolic-cap and dimpled solutions, but instead there are smooth transitions as $c_{\text {out }}$ is varied. Further, there are ranges of $c_{\text {out }}$ for which no parabolic-cap solutions exist. This is a consequence of there being very large hydration stresses near the edges of the vesicle where $h$ is very small. These hydration stresses are balanced by bending stresses at the edges, which perturb the global solution. These perturbations excite global bending modes of the membrane, and the smooth transitions between parabolic-cap and dimpled solutions correspond to a rapid increase in the amplitude of
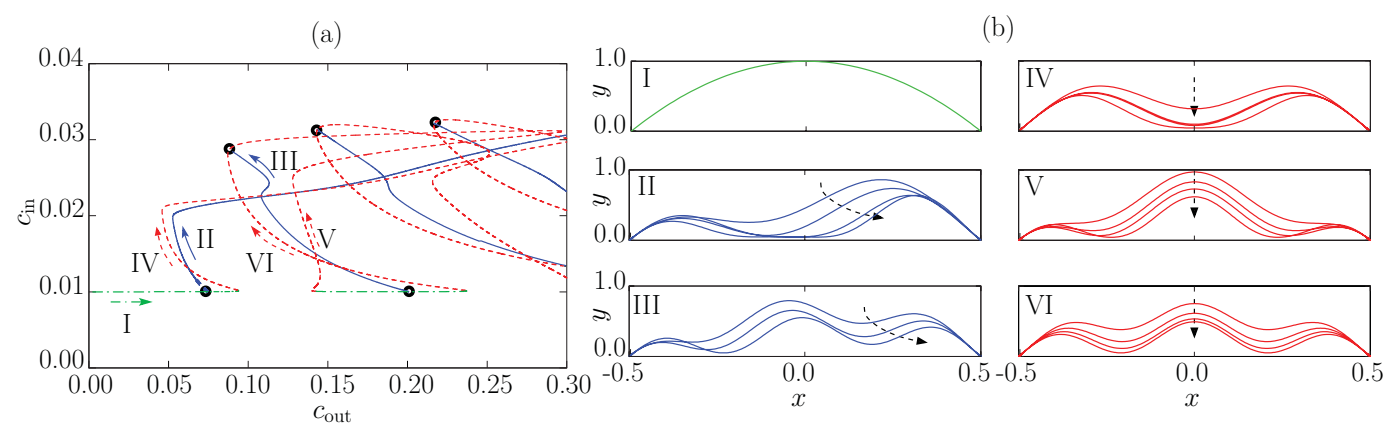

FIG. 4. (Color online) Equilibrium solutions for Problem I, with $P_{0}=10^{8}$ and $\lambda=5 \times 10^{-3}$. The other parameter values are the same as in Fig. 3. (a) The variation of internal sugar concentration $c_{\text {in }}$ as the external sugar concentration $c_{\text {out }}$ is varied. Circles denote bifurcation points, and arrows denote the variation of the membrane shapes [shown in (b)] as the branch is traversed (see text). (b) Equilibrium membrane shapes. Arrows show the direction in which the branches are traversed in (a) and solutions are plotted for $c_{\text {in }}=0.015$ and multiples of 0.005 thereafter. 
the bending mode. These transitions approach the bifurcation points in Fig. 4(a) as either $P_{0}$ or $\lambda$ is decreased. Because the boundary-layer regions correspond to $P_{0} \exp (-h / \lambda)=O(1)$, we conclude that the discontinuity of the parabolic-cap branch is caused by boundary-layer effects of hydration stresses. We note that this effect is a somewhat unphysical consequence of the clamped boundary conditions prescribed; as described in Sec. II B 1, the long-wave approximation does not allow the behavior of the membrane near the contact point to be fully resolved. A more detailed analysis of the behavior of the membrane in these regions falls outside the scope of this paper.

\section{Problem II}

Figures 5(a)-5(c) show how $c_{\text {in }}$ and $\Gamma$ vary as $c_{\text {out }}$ is varied for the isolated blister problem, and Fig. 5(d) shows how the membrane shape varies as $c_{\text {out }}$ is increased. The zero-slope condition prescribed for this problem prohibits the parabolic-cap solution observed previously. Instead, if $c_{\text {out }} \approx c_{\text {init }}$ then the only possible equilibrium solution is a symmetric blister, whose shape is somewhat analogous to those depicted in Fig. 4(bV). As for Problem I, the volume of liquid beneath the membrane decreases and the membrane's compression $\Gamma$ increases as $c_{\text {out }}$ is increased. In the absence of hydration stresses, there is a subcritical bifurcation toward asymmetric membrane shapes at the point where $\Gamma$ is a root of the square-bracketed expression in Eq. (35). As for Problem I, at this value of $\Gamma$ the membrane's compression is compatible with an asymmetric bending mode that satisfies both the slope constraint (32b) and the height constraint (33b). Further, as $c_{\text {out }}$ continues to be increased, the membrane's compression must remain constant on the asymmetric solution branch. This compression supports the osmotic pressure difference across the membrane, and so $c_{\text {in }}-c_{\text {out }}$ approaches a constant value as $c_{\text {out }}$ is increased. Along the symmetric solution branch, the membrane's compression increases as $c_{\text {out }}$ is increased, which corresponds to the membrane's wrinkles having shorter wavelength [see Fig. 5(dI)]. However, the wavelength of these wrinkles is bounded in the absence of hydration stresses, and the membrane's compression has the bound $\Gamma \approx 130$ shown in Fig. 5(b). This bound is an unphysical consequence of the membrane penetrating the substrate. To see this, we substitute the boundary constraints (32a) and (33a) into the volume constraint (31) to obtain

$$
A\left[\left(\frac{2}{\sqrt{\Gamma}}-\frac{\sqrt{\Gamma}}{6}\right) \sin \left(\frac{\sqrt{\Gamma}}{2}\right)-\cos \left(\frac{\sqrt{\Gamma}}{2}\right)\right]=\frac{2 c_{\mathrm{init}}}{3 c_{\mathrm{in}}} .
$$

The square-bracketed expression approaches zero as $\Gamma \rightarrow 133$ owing to the negative contributions to the vesicle's volume from the regions where $h<0$. Hence, in this limit, the bending amplitude $A$ would increase with $\Gamma$, but is prevented from doing so indefinitely by the area constraint (30). Instead, $c_{\text {in }}$ increases without limit and $\Gamma$ approaches 133 as $c_{\text {out }}$ is increased.

As for Problem I and as shown in Fig. 5(a), the main effect of reintroducing hydration stresses is that the removal of water is impeded as $c_{\text {out }}$ is increased. Figure 5(b) shows how, for larger values of $c_{\text {out }}$, the membrane's compression is increased by the presence of hydration stresses, owing to its being pressed in opposing directions by the osmotic pressure difference across the membrane and the hydration stresses. There are (a)

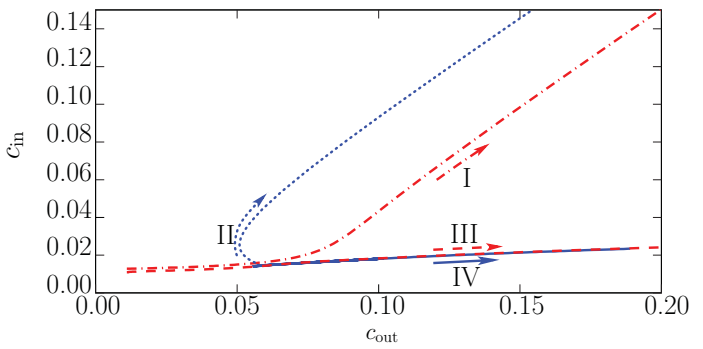

(c)

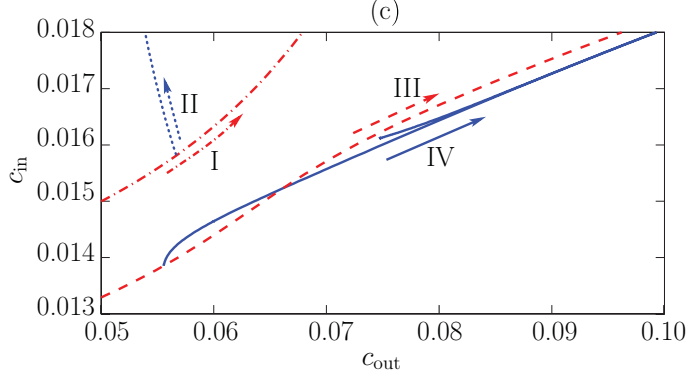

(b)

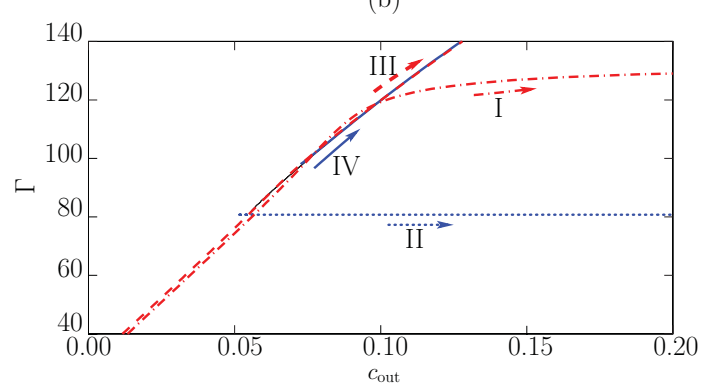

(d)
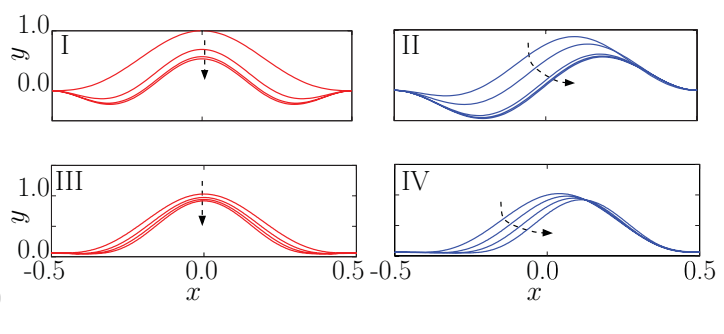

FIG. 5. (Color online) Equilibrium solutions for Problem II, in the absence (dash-dotted, labeled I, and dotted, labeled II) and presence (dashed, labeled III, and solid, labeled IV) of hydration stresses. Parameter values used are the same as for Fig. 4. (a) The variation of internal sugar concentration $c_{\text {in }}$ as the external sugar concentration $c_{\text {out }}$ is varied. The symmetric solution branches are labeled I and III, and the asymmetric solution branches are labeled II and IV. (b) The variation of the membrane's compression $\Gamma$ as $c_{\text {out }}$ is varied. (c) The same plot as (a) except with a narrower range of values for $c_{\text {in }}$ and $c_{\text {out }}$ so that the bifurcation points may be seen more clearly. (d) Equilibrium membrane shapes in the absence (I and II) and presence (III and IV) of hydration stresses. Arrows show the direction in which the branches are traversed, and solutions are shown for $c_{\text {out }}=0.08$ and multiples of 0.02 thereafter. 

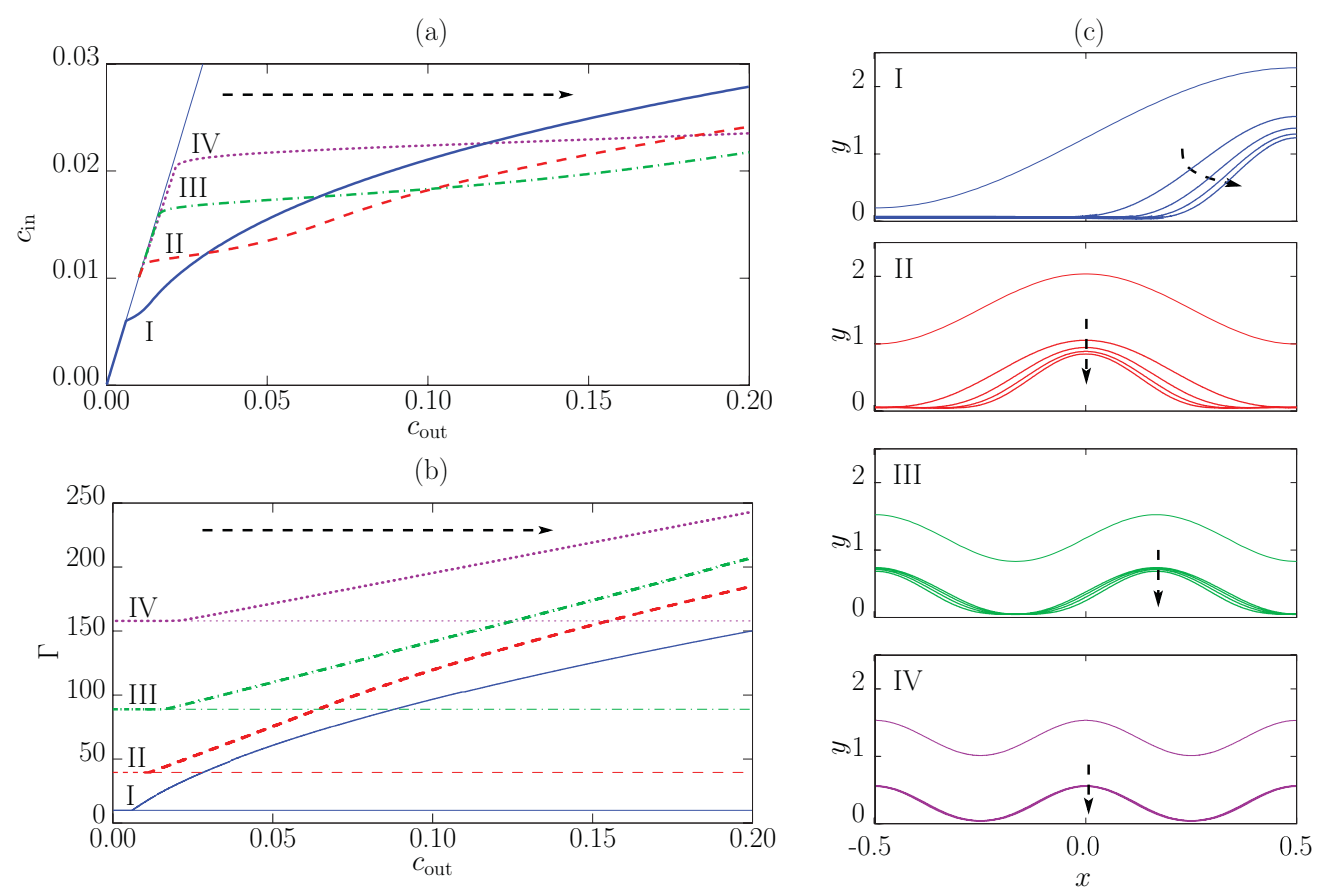

FIG. 6. (Color online) Equilibrium solutions for Problem III, in the absence (thin lines) and presence (bold lines) of hydration stresses. Parameter values used are the same as for Fig. 4. (a) The variation of internal sugar concentration $c_{\text {in }}$ as the external sugar concentration $c_{\text {out }}$ is varied. (b) The variation of membrane compression $\Gamma$ as the external sugar concentration $c_{\text {out }}$ is varied. (c) Equilibrium membrane shapes, in the absence (thin lines, and offset in the $y$ direction) and presence (bold lines) of hydration stresses, for each of the four modes shown in (a) and (b). Arrows show the direction in which the solution branches are traversed, and the solutions are shown at $c_{\text {out }}=0.05$ and multiples of 0.05 thereafter. For these parameter values, the membrane's shape for mode IV are almost indistinguishable from each other.

again several bifurcations from the symmetric solution branch toward asymmetric solutions, which can be seen most easily in Fig. 5(c). In this problem, these bifurcations are an artifact of the truncation of the domain. One might expect that on an infinite domain, all equilibrium solutions are identical up to a translation. Away from the blister, the membrane height approaches the value $h^{*}$ for which the osmotic stress balances the hydration stress [and which solves (26a)]. Linearization of the shape equation (28) about this value implies that the membrane's height converges toward $h^{*}$ with oscillations that decay exponentially away from the blister. However, owing to the imposition of boundary conditions at a finite distance from the blister, the shapes of the computed solutions are slightly different to the shape of an isolated blister on an infinite domain. Corrections to this shape are, therefore, needed near the boundaries to satisfy (26), and as $c_{\text {out }}$ is increased there are several supercritical bifurcations that occur when these corrections may be achieved by way of asymmetric corrections. The asymmetric states differ only very slightly from the symmetric ones, as shown by the close correspondence between the values of $c_{\text {in }}$ and $\Gamma$ in Figs. 5(a)-5(c). As $c_{\text {out }}$ is increased, the volume of the blister decreases, and there is a longer wetting layer separating it from the boundaries. The boundary conditions (26) therefore provide a better representation of an isolated blister as $c_{\text {out }}$ is increased. Additional calculations, not shown here, suggest that the effect of varying $P_{0}$ and $\lambda$ is primarily to modify the thickness $h^{*}$ of the wetting layer, whereas the value of $c_{\text {in }}$ is not appreciably affected. A decrease in $\lambda$ has the additional effect of decreasing the amplitude of the oscillations of $h$ about $h^{*}$ at the edges of the dimple, which is a consequence of the increased sensitivity of hydration stresses to $h$ for smaller values of $\lambda$.

\section{Problem III}

Figures 6(a) and 6(b) show how $c_{\text {in }}$ and $\Gamma$ vary with $c_{\text {out }}$ for the "many-blister" problem, and Fig. 6(c) shows how the membrane shape varies as $c_{\text {out }}$ is increased. As described in Sec. III A, solutions fall onto one of several discrete branches in the absence of hydration stresses. In contrast to the previous two problems, these branches do not intersect and there are no bifurcations as $c_{\text {out }}$ is increased. Instead, the membrane's compression $\Gamma$ remains constant and the internal sugar concentration $c_{\text {in }}$ remains equal to $c_{\text {out }}$. The membrane's shape varies only by a vertical translation as necessary to satisfy the volume constraint (23d).

When hydration stresses are present, the membrane's behavior passes through three distinct regimes as $c_{\text {out }}$ is increased. For small values of $c_{\text {out }}$, the membrane's height is large enough that the hydration stresses have a negligible effect on it, and its shape in this regime is indistinguishable from those obtained in the absence of hydration stress. For moderate values of $c_{\text {out }}$ there is a second regime that is relevant when the membrane begins to approach the substrate. In this regime, hydration stresses are significant and repel the membrane from the substrate as $c_{\text {out }}$ is increased, thereby impeding the removal of water. This is shown most clearly in Fig. 6(a) for modes II-IV, where for each mode there is an abrupt change in the gradient of $c_{\mathrm{in}}$, as $c_{\text {out }}$ is varied, from unity to a significantly 
smaller value. Figure 6(b) shows how the hydration stress causes the membrane's compression to increase with the sugar concentration $c_{\text {out }}$ outside the membrane. For larger values of $c_{\text {out }}$ the equilibrium solutions fall into a third regime, which is shown most clearly in Figs. 6(a) and 6(c) for modes I and II. In this regime, a significant portion of the membrane is pressed flat against the substrate to form a wetting layer. The extent of this wetting layer increases with $c_{\text {out }}$, and the slopes of the sides of the blister must also increase to satisfy the global area constraint (23c). The primary effect of increasing $c_{\text {out }}$ in this regime is to deform the walls of the blister further, with only a modest increase in the extent of the wetting layer. For the parameter values used, the energetic cost of deforming the blister in this way is smaller than the cost of overcoming the hydration stresses and, as shown in Fig. 6(a) for modes I and II, the rate of increase of $c_{\text {in }}$ with $c_{\text {out }}$ is slightly greater than in the intermediate regime. As for Problem II, the primary effect of varying the values of $P_{0}$ and $\lambda$ is to change the thickness of the wetting layer. A decrease in the value of $\lambda$ again has the additional effect of "flattening" the membrane at the edges of the blister, so that oscillations of the membrane's height there are suppressed.

We have considered equilibrium membrane shapes for three distinct sets of boundary conditions that are intended to represent three distinct drying processes. We found that for Problems I and II there is only one symmetric solution for small values of the external sugar concentration $c_{\text {out }}$, and that there are bifurcations from these solutions toward asymmetric membrane shapes as $c_{\text {out }}$ is increased. For Problem I, in the absence of hydration stresses there are additional bifurcations toward symmetric dimpled shapes. In the presence of these stresses the bifurcations are instead smooth transitions between parabolic-cap shapes and dimpled shapes. For Problem III there are no bifurcations, and the solutions instead fall onto one of several disconnected branches, each of which represent there being a different number of blisters within the domain. There are a countably infinite number of such branches, which are valid for any value of $c_{\text {out }}$.

In the following section we consider the stability of the equilibrium solutions obtained here, with the aim of determining which of them is dynamically most favorable.

\section{STABILITY ANALYSIS OF THE STEADY STATES}

We disturb the equilibrium states as follows:

$$
\begin{aligned}
h(x, t) & =\bar{h}(x)+\widehat{h}(x) e^{\sigma t} \\
c_{\text {in }}(t) & =\bar{c}_{\text {in }}+\widehat{c}_{\text {in }} e^{\sigma t} \text { and } \quad \Gamma=\bar{\Gamma}+\widehat{\Gamma} e^{\sigma t},
\end{aligned}
$$

where we denote steady-state variables with an overbar and the amplitudes of the disturbance with a hat. Substitution of (37) into (23) and keeping only the terms that are linear in the disturbance amplitude yields

$$
\begin{aligned}
\sigma \widehat{h} & =\left(\frac{\bar{h}^{3}}{12} \widehat{p}_{x}\right)_{x}+k_{1} \widehat{c}_{\text {in }}-k_{2} \widehat{p}, \\
\widehat{p} & =\widehat{h}_{x x x x}+\bar{\Gamma} \widehat{h}_{x x}+\widehat{\Gamma} \bar{h}_{x x}+\frac{P_{0} \widehat{h}}{\lambda} \exp (-\bar{h} / \lambda),
\end{aligned}
$$

subject to the constraints

$$
\begin{aligned}
& \int_{-1 / 2}^{1 / 2} \bar{h}_{x} \widehat{h}_{x} d x=0, \\
& \bar{c}_{\text {in }} \int_{-1 / 2}^{1 / 2} \widehat{h} d x+\widehat{c}_{\text {in }} \int_{-1 / 2}^{1 / 2} \bar{h} d x=0 .
\end{aligned}
$$

The boundary conditions for each problem are obtained through linearizing the boundary conditions (25)-(27) for the time-dependent problem, and are given by

$$
\widehat{p}_{x}=0 \quad \text { at } \quad x= \pm \frac{1}{2},
$$

together with

$$
\left.\begin{array}{ll}
\text { Problem I: } & \widehat{h}=\widehat{h}_{x}=0 \\
\text { Problem II: } & \widehat{h}-\frac{k_{1} \lambda}{k_{2} P_{0}} e^{\bar{h} / \lambda} \widehat{c}_{\text {in }}=\widehat{h}_{x}=0 \\
\text { Problem III: } & \widehat{h}_{x x x}=\widehat{h}_{x}=0
\end{array}\right\} \text { at } x= \pm \frac{1}{2} .
$$

These are two-point boundary-value problems with eigenvalues $\sigma$. In Appendix B we describe how estimates of these eigenvalues may be computed using a finite-difference method. We find that better-resolved estimates may be obtained by instead calculating the eigenmodes using a continuation method [25] that has previously been implemented using AUTO-07P [26,27] for other linear stability problems. The starting solution for this method is a steady state, calculated as described in Sec. III, together with the trivial eigenmode (for which all disturbance variables are zero). The growth rate $\sigma$ is initialized with an approximate value obtained using the finite-difference method described in Appendix B. The homogeneous boundary condition (39c) is replaced by

$$
\widehat{h}_{x}=\beta_{ \pm} \quad \text { at } \quad x= \pm \frac{1}{2},
$$

where the values of $\beta_{+}$and $\beta_{-}$are initially zero. The eigenmode is then forced to be nontrivial by adjusting one or both of the parameters $\beta_{ \pm}$to some nonzero value (e.g., 1), while holding $\sigma$ fixed. After this stage we have a nontrivial solution to (38) which satisfies all boundary conditions except (39c). We must, therefore, reimpose this boundary condition by gradually adjusting the values of $\beta_{ \pm}$back to zero while keeping the solution nontrivial. This is achieved by prescribing the additional constraint that some measure of the eigenmode's magnitude remains nonzero, for example, by imposing the condition

$$
\int_{-1 / 2}^{1 / 2} \widehat{h}^{2} d x=\beta_{0}, \quad \beta_{0} \neq 0 .
$$

As we reimpose the boundary condition (39c), we allow the growth rate $\sigma$ to vary freely while keeping $\beta_{0}$ fixed. We, thereby, obtain a nontrivial disturbance eigenmode which satisfies all boundary conditions and which has an associated growth rate $\sigma$. We may then adjust the value of $c_{\text {out }}$ while allowing $\sigma$ to vary. There are several distinct eigenmodes, each of which has a different growth rate; the particular eigenmode that is obtained using this method depends strongly on the initial estimate of the eigenvalue $\sigma$ and on the way in which the homogeneous boundary condition (40) is initially modified. We, therefore, compute rough estimates of the eigenmodes 

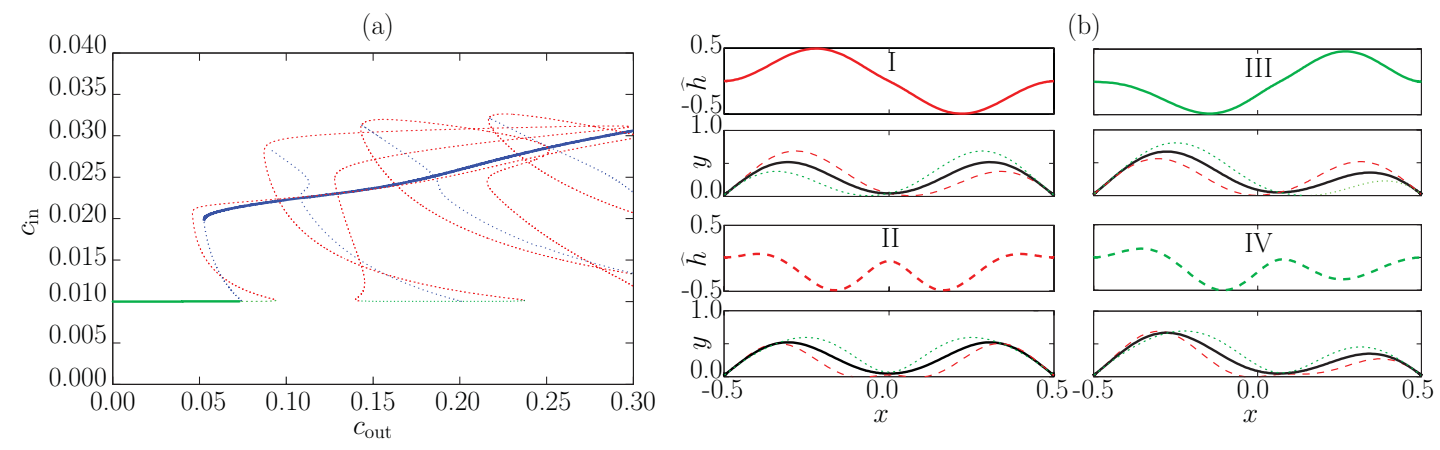

FIG. 7. (Color online) The stability of equilibrium solutions and the height profiles of disturbance eigenmodes for Problem I. Parameter values used are the same as for Fig. 4 , and the height profiles are plotted for $c_{\text {out }}=0.1$. (a) The bifurcation diagram shown in Fig. 4 with the stable solutions shown using bold, continuous lines. Unstable solutions are shown using dotted lines. (b) The amplitude $\widehat{h}$ of the disturbance (above) and the perturbed membrane shape (beneath). The amplitudes have been normalized to have a maximum magnitude of 0.5 , and the perturbed membrane shapes are a superposition of the shape of the steady state (shown by a solid line) and \pm 0.4 times the disturbance mode.

using the finite-difference method described in Appendix B and use these eigenmodes to guide our initial guess for $\sigma$ and the nature (i.e., symmetric, antisymmetric, or neither) of the perturbation to the boundary condition (40).

\section{A. Results}

As described in Sec. II B 1, the evolution equation (23a) has essential singularities at the points where $h=0$. For simplicity we, therefore, only consider the stability of the steady states for which hydration stresses (which prevent the membrane from touching down onto the substrate) are present. In this case, the presence of a wetting layer ensures that $h>0$ everywhere for all problems, with the exception of the edges of the domain for Problem I.

\section{Problem I}

Figure 7(a) shows the stability properties of the equilibrium solutions calculated in Sec. III B 1, and the two most unstable eigenmodes are shown for the symmetric and asymmetric equilibrium solutions, which have $c_{\text {out }}=0.1$, in Fig. $7(\mathrm{~b})$. The parabolic-cap solution branch is stable to all perturbations when the external sugar concentration $c_{\text {out }}$ is smaller than the critical value $c_{\text {out }}^{*}$ that corresponds to the first bifurcation toward asymmetric solutions. If $c_{\text {out }}>c_{\text {out }}^{*}$, then the paraboliccap solutions are unstable to an antisymmetric disturbance whose shape is shown in Fig. 7(bI) for $c_{\text {out }}=0.1$. As $c_{\text {out }}$ is increased, other perturbation modes become unstable as successive bifurcation points are passed. For example, when $c_{\text {out }} \gtrsim 0.14$, the eigenmode depicted in Fig. 7(bII) is unstable, and when $c_{\text {out }} \gtrsim 0.2$ there is a third unstable, symmetric eigenmode. It follows that, for an initially parabolic cap for which $c_{\text {out }}$ is much larger than $c_{\text {in }}$, the evolution of the shape is extremely sensitive to perturbations. We anticipate that a time-dependent calculation would show that, as water is drawn out of the vesicle, disturbances to the membrane's shape grow until $c_{\text {in }}-c_{\text {out }} \sim k_{2} p / k_{1}$. (As noted in Appendix A, the value of the ratio $k_{2} / k_{1}$ used here is much larger than observed experimentally; the values obtained here for the concentration differences across the membrane are also, therefore, much larger.)
When $c_{\text {in }}$ eventually approaches $c_{\text {out }}$, the membrane is likely to be wrinkled. In Sec. IV A 3 we show that a wrinkled membrane tends to coarsen as time progresses. We therefore restrict attention here to the steady states that have at most one dimple [whose shapes are depicted in Fig. 4(bI, II, and IV)] and conclude that the steady states with a larger number of dimples [e.g., Fig. 4(bIII, V, and VI)] are unstable to coarsening.

The stability analysis shows that, for the symmetric dimpled solutions where the dimple is not close to the substrate, there are two unstable eigenmodes, one of which is an antisymmetric disturbance [Fig. 7(bI)] and the other of which is an "undimpling" disturbance [Fig. 7(bII)]. The superposition of these disturbances would represent the movement of the dimple from the center of the vesicle to an off-centered position and the adjustment of $c_{\text {in }}$ toward the value that corresponds to the asymmetric equilibrium solution. However, if the equilibrium solution has its dimple pressed against the substrate, then hydration stresses cause the membrane to form a wetting layer in the center and the liquid is largely confined to lobes on either side. In this case, the undimpling disturbance mode is stable, whereas the antisymmetric mode remains unstable and now represents the transfer of water from the lobe on one side of the vesicle to the other lobe. This transfer can take place by way of mass transfer through the thin wetting layer, by way of flux out through the membrane of one lobe and in through the membrane of the other lobe, or through a combination of both. For both mechanisms, the growth (or shrinkage) of one lobe results in an increase (or decrease) in its height, which means that the curvature of its membrane also increases (decreases) and the pressure inside the lobe falls (rises) owing to the membrane's compression. The pressure difference between lobes then drives further transfer of fluid from the shrinking lobe to the growing lobe. Hence, both mechanisms reinforce the growth of one lobe at the expense of the other. The transfer of fluid through the wetting layer is somewhat analogous to the coarsening instability observed in an array of liquid droplets connected through conduits [28]. However, in that problem the pressure drop across the interface of a larger droplet is due to the decrease (rather than increase) in its curvature as its volume is increased, which causes a pressure drop owing to the tension (rather than compression) of 

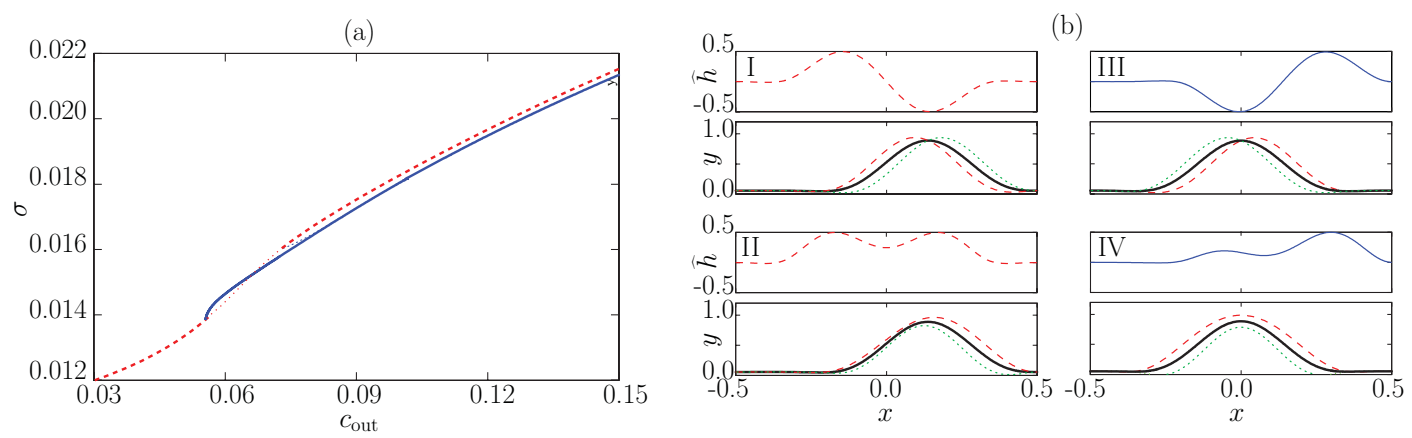

FIG. 8. (Color online) The stability of equilibrium solutions and the height profiles of disturbance eigenmodes for Problem II. Parameter values used are the same as for Figs. 4 and 5, and the height profiles in (b) are plotted for $c_{\text {out }}=0.15$. (a) The bifurcation diagram shown in Fig. 5 with the stable solutions shown using bold lines. Unstable solutions are shown using thin dotted lines and can be seen for $0.055 \leqslant c_{\text {out }} \leqslant 0.075$. (b) The amplitude $\widehat{h}$ of the disturbance (above) and the perturbed membrane shape (beneath). The amplitudes have been normalized to have a maximum magnitude of 0.5 , and the perturbed membrane shapes are a superposition of the shape of the steady state (shown by a solid line) and \pm 0.4 times the disturbance mode.

the surface. Another difference is that the problem analyzed in Ref. [28] does not involve mass transport across the interface, whereas here water can be gained or lost through the membrane owing to pressure differences across it. However, the relative importance of pressure-driven flux through the membrane compared to the flux through the wetting layer depends on the size of $k_{2}$; for very small values of $k_{2}$ a pressure drop between lobes will predominantly drive a flow through the wetting layer rather than through the membrane.

The asymmetric equilibrium solutions take one of two qualitatively different shapes depending on whether the membrane interacts with the substrate. The small range of $c_{\text {out }}$ for which this steady state is unstable corresponds to $c_{\text {in }}<0.02$ in Fig. 7(a), and for these parameter values there is negligible interaction between the membrane and the substrate. For these values there is a single unstable disturbance mode that represents water being drawn into or out of the vesicle depending on the way in which the vesicle is initially disturbed. The vesicle may either grow, so that its shape approaches that of a parabolic cap, or shrink, so that its shape approaches an asymmetric dimpled shape for which the dimple is pressed flat against the substrate. When $c_{\text {in }}>0.02$, the asymmetric steady-state solution is stable to all disturbances. This solution has two lobes of different sizes that are separated by a wetting layer, and it is the only stable solution when $c_{\text {out }}>0.07$.

\section{Problem II}

Figure 8(a) shows the stability properties of the equilibrium solutions calculated in Sec. III B 2, and the two most unstable eigenmodes are shown (for equilibrium solutions with $c_{\text {out }}=$ 0.15 ) in Fig. 8(b). The symmetric steady state is typically stable, except for a narrow window that is given by $0.055 \lesssim$ $c_{\text {out }} \lesssim 0.075$. In this range there is a significant interaction between the blister and the boundary, owing to the volume of the blister being too large to justify its treatment as an isolated blister. It is likely that the observed instability is a consequence of this interaction. For larger values of $c_{\text {out }}$, which more accurately represent the situations in which the blister is isolated, both the symmetric and the asymmetric equilibrium solutions are stable to all disturbances. The most unstable eigenmodes are depicted in Fig. 8(bI,III) and represent a translation of the blister. The growth rate remains negative, but its magnitude decreases as $c_{\text {out }}$ is increased. The approach of this eigenmode toward neutral stability corresponds to the limiting case where there is no interaction between the blister and the boundary. In this limit, the neutrally stable eigenmode represents translation at a constant speed of the blister along the wetting layer.

For both symmetric and asymmetric steady states, the second of the two most unstable eigenmodes represents growth or shrinkage of the blister. The dimpled shapes of the eigenmodes suggest that if this eigenmode were unstable, the blister would eventually split into two blisters or alternatively attain a shape similar to one of those shown in Fig. 4(bVI) for Problem I. This eigenmode is always stable, which suggests that the dynamically favored membrane configuration is the one that minimizes the number of distinct blisters. We build on this conclusion in the following section where we consider the interaction among multiple blisters.

\section{Problem III}

Figure 9(a) shows the stability properties of the equilibrium solutions calculated in Sec. III B 3, and Fig. 9(b) shows the most unstable eigenmode for the equilibrium solutions with $n=\mathrm{I}-\mathrm{III}$. The modes for which $n=\mathrm{III}$ and IV are unstable to a disturbance mode that represents a coarsening instability. As shown in Fig. 9(bIII), this instability involves the transfer of fluid between neighboring blisters. With the exception of the mode given by $n=$ IIA, in regimes where the membrane is close to the substrate, the growth rates of all eigenmodes increase in magnitude as $c_{\text {out }}$ increases. The reason is that the stability of the membrane is determined by a balance between the osmotic pressure, the membrane compression, and the hydration stress that is exerted on the membrane, which all increase in magnitude as $c_{\text {out }}$ is increased. The strength of the stabilizing or destabilizing mechanism therefore increases with $c_{\text {out }}$, leading to a much faster growth (or decay) of perturbations. When $c_{\text {out }}$ is small, the growth rate of perturbations to modes $n=$ III and IV are very large. In this regime there is no wetting layer, and there is, therefore, 
(a)

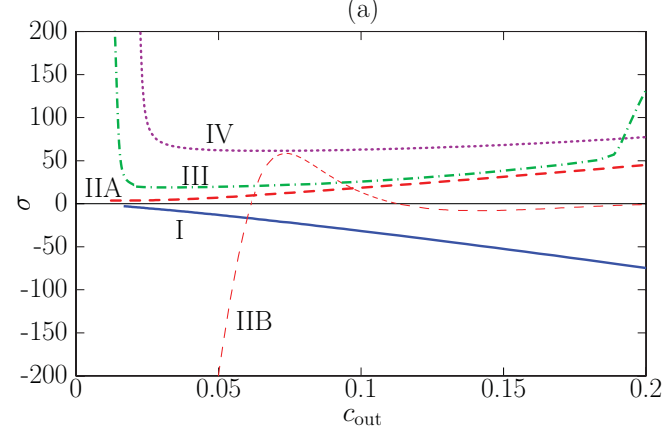

(b)

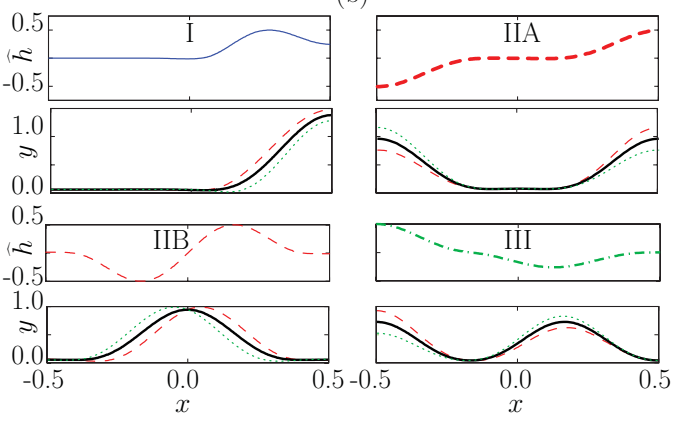

FIG. 9. (Color online) Growth rates and height profiles of disturbance eigenmodes for Problem III. Parameter values used are the same as for Fig. 4, and the height profiles are plotted for $c_{\text {out }}=0.1$. (a) The growth rates of the most unstable disturbance mode for each of the four steady states given by $n=\mathrm{I}, \ldots, \mathrm{IV}$. Two growth rates are shown for mode II because the stability properties of this mode depend on where the symmetry boundary conditions are prescribed (see text). (b) The amplitude $\widehat{h}$ of the disturbance (above) and the perturbed membrane shape (beneath). The amplitudes have been normalized to have a maximum magnitude of 0.5 , and the perturbed membrane shapes are a superposition of the shape of the steady state (shown by a solid line) and \pm 0.4 times the disturbance mode.

a much smaller viscous resistance to fluid flow beneath the membrane.

The steady state given by $n=\mathrm{I}$ is stable for all values of $c_{\text {out }}$ explored here. Fluid transfer between lobes, which was the most unstable disturbance for the steady states with $n=\mathrm{III}$ and IV, is prohibited in this case by the symmetry constraint. The most unstable eigenmode instead represents growth and, eventually, division of the lobe into two daughter lobes. This disturbance is stable for all values of $c_{\text {out }}$, and the decay rate (given by $-\sigma$ ) increases with $c_{\text {out }}$, which is a consequence of the larger restoring effects of osmotic pressure and hydration stresses.

There are two permissible equilibrium solutions for the case $n=\mathrm{II}$, which differ only by a translation of $\frac{1}{2}$ in the $x$ direction. Whereas the steady-state solution is unaffected by this translation, the time-dependent motion is significantly different in either case owing to the boundary conditions prescribed. The mode depicted in Fig. 9(bIIA) represents a periodic array (of period 2) of blisters, for which the shapes of neighboring blisters are only indirectly related through dynamic interactions. In contrast, the mode shown in Fig. 9(bIIB) instead represents a periodic array of blisters, for which the shapes of neighboring blisters are directly related through the constraint that that they be the mirror image of each other. The former case permits the transfer of fluid between adjacent blisters and is thus unstable to a coarsening instability in an analogous way to the modes with $n \geqslant$ III. However, in the latter case the transfer of fluid between blisters is prohibited, and so there is no such coarsening instability. Instead, the most unstable disturbance represents a translation of the blisters along the wetting layer. This disturbance mode is somewhat analogous to that of isolated blisters in Problem II, in that there is a narrow range of $c_{\text {out }}$ for which it is unstable. Within this range, the volume of the blister is large and there is only a short wetting layer that separates adjacent blisters. The symmetry constraint, here, means that the translation eigenmode represents successive pairs of blisters moving toward each other and coalescing. As for Problem II, as $c_{\text {in }}$ is increased, the distance between the blister and the boundaries is greater and the eigenmode more closely represents the translation of an isolated blister along the wetting layer. The interaction between neighboring blisters is diminished owing to their separation by a long wetting layer, and the growth rate of this mode again approaches zero.

In this section we have considered the stability of the equilibrium shapes obtained for each of the three problems described in Sec. II B. In Problem I we found that a vesicle favors a parabolic-cap shape for sufficiently small values of $c_{\text {out }}$, but for larger values of $c_{\text {out }}$ the membrane is unstable to buckling and instead favors an asymmetric dimpled shape. In Problem II it was shown that an isolated blister on a thin wetting layer is typically stable. Whereas parameter ranges exist for which these blisters are unstable, the instability in these cases may be attributed to the interference between the blister and the clamped conditions prescribed at the boundaries. In these situations, the proximity of the boundaries to the blister means that it is inaccurate to refer to such blisters as isolated. The analysis of Problem III demonstrates that, for all external sugar concentrations, a wrinkled membrane will tend to coarsen toward a configuration that is less wrinkled, thereby justifying the restriction of our analysis of Problem I to vesicle shapes with few dimples.

\section{DISCUSSION}

We have developed a model that describes the behavior of a vesicle during drying processes and, through prescription of appropriate boundary conditions, we have shown how our model may also be applied to describe the behavior of a membrane as the underlying fluid is removed through drying. Our model, derived using a long-wave approximation, represents a significant simplification of the problem and nonetheless encapsulates the effects of water transport across the membrane and of the interaction between the membrane and the fluid flow beneath it. We have shown how these effects can give rise to a rich bifurcation structure when the edges of the membrane are clamped and how the equilibrium configurations of the membrane can vary significantly depending on the boundary conditions that are prescribed.

The stability analysis of the equilibrium solutions has yielded several interesting results. The analysis of Problem III showed that a membrane that has several undulations will 
tend to coarsen toward a shape that has fewer undulations. We postulate that because the membrane's compression $\Gamma$ may be thought of as a measure of the number of undulations of the membrane, and because the surface energy increases with $\Gamma$, the configurations favored by the membrane typically have fewer undulations. This conclusion is consistent with the stability analysis for Problem II, which showed that an isolated blister is stable to all perturbations. The tendency of buckled, compressed states to form fewer undulations has also been observed [29] in the context of surface instabilities of a compressed, semi-infinite, elastic solid, for which wrinkles are susceptible to a coarsening instability whereby a single "crease" is formed in the solid. Another interesting result found here is the tendency of a clamped vesicle to favor an asymmetric steady state despite the application of symmetric initial and boundary conditions. This may also be interpreted as a consequence of energy minimization, as the membrane compression associated with an asymmetric configuration (with one dimple) is smaller than that associated with a symmetric dimpled configuration.

As stated throughout this paper, the value of $k_{2}$ used in our calculations is much larger than typically encountered in experiment. The reason for our use of such large values was primarily to facilitate computation (and presentation) of numerical results and also to focus on the later times during the desiccation process when the concentrations inside and outside the vesicle are almost equal. We may now draw together the conclusions of the preceding sections to describe what happens at earlier times in the desiccation process. Suppose that the vesicle is initially a parabolic cap, and that the surrounding solution has a lower sugar concentration than the concentration $c_{\text {init }}$ inside the vesicle. The membrane will, at that moment, be under tension in order to balance the osmotic pressure difference. If the external solution is then dried so that $c_{\text {out }}$ increases to a value that is larger than $c_{\text {init }}$, then because $k_{2}$ is very small there is a transient motion in which water is rapidly drawn out of the vesicle and, as described in Sec. IV A 1, the parabolic cap is unstable to many bending modes. Hence, during this transient phase, the wrinkling is likely to take place on very short length scales. This transient phase ends when $c_{\text {in }}$ is almost equal to $c_{\text {out }}$, at which time the analysis of our model may be applied. From our analysis of Problem III in Sec. IV A 3, any small-scale wrinkles that are present will coarsen on a time scale that decreases with their length scale. This coarsening behavior, therefore, represents a second transient motion in which the vesicle approaches a shape with at most one dimple. The final approach of the vesicle toward a stable asymmetric equilibrium was analyzed in Sec. IV A 1 and takes place on a much longer time scale.

One unsatisfactory aspect of our model is that the long-wave approximation breaks down near the edges of the vesicle. This means that, whereas our model should be valid in the interior of the vesicle, our estimation of the boundary conditions should be improved for the situation where a vesicle is attached to a flat substrate. Efforts are currently under way to resolve the behavior of the vesicle in such regions. Similarly, the prescription of clamped boundary conditions to represent an isolated blister was shown to exhibit unphysical stability properties owing to the distortion of the blister when it is close to one of the boundaries. This may, perhaps, also be rectified through application of appropriate matching conditions that would represent the decay of undulations in the film away from the blister.

The model described here contains many of the important effects that arise during desiccation processes, but there are several ways in which our model may be extended. For instance, the solution inside (or beneath) the membrane has been assumed to be homogeneous. Whereas this is a valid assumption on the small length scales associated with biological cells, it is unlikely to remain valid on larger length scales. A possible extension of our model would therefore be to allow the concentration of the solution to vary spatially. Large concentration gradients may also arise during the transient phase where water is rapidly removed through the membrane, and it might be the case that these gradients can give rise to some interesting secondary effects through modification of the physical properties (such as the viscosity) of the solution. In the context of the drying of biological cells, one of these effects is the phase transition of a sugar solution to a glassy phase above a critical sugar concentration. Diffusion of water through this phase is much slower than through an aqueous solution, and so it is possible that the glassy phase might form a "skin" near the surface of the vesicle, thereby slowing the desiccation process.

In our model we have treated the membrane as an elastic shell, and possible extensions to our model lie in a more realistic treatment of the membrane. Whereas our assumption that the membrane is incompressible is justified by its very small resistance to bending compared with its resistance to stretching [3], membranes have been observed to change area when placed under large tensions. A possible avenue for further work therefore lies in allowing the membrane's area to change locally in addition to its tension and to relate the two by an equation of state. This equation of state would represent a simple modification to our model and may be a fruitful avenue for further work.

\section{ACKNOWLEDGMENTS}

We thank Peter S. Stewart, Michael B. Gratton and Michael J. Davis for helpful discussions. This work was supported by the National Institutes of Health through Grant No. 5R01GM086886.

\section{APPENDIX A: ESTIMATION OF PARAMETER VALUES}

Here we estimate values of physical parameters that are representative of experiments that concern the desiccation of cells and vesicles and justify the approximations made when deriving the model equations in Sec. II. We summarize the values of the dimensional quantities and dimensionless parameters in Table I.

Experiments have been performed in which the shapes of vesicles are controlled by means of chemically patterning the substrate on which it lies [6]. Very small aspect ratios have been achieved, and it is in these situations that it is appropriate to use the lubrication approximation. We estimate the vesicle's dimensions by assuming that it is initially a parabolic cap 
TABLE I. Typical values of the dimensional parameters encountered in the desiccation of biological cells, and approximate values of the dimensionless parameters that are used in the main text.

\begin{tabular}{|c|c|c|c|c|c|}
\hline $\begin{array}{l}\text { Dimensional } \\
\text { quantity }\end{array}$ & Typical value & $\begin{array}{c}\text { Dimensionless } \\
\text { parameter }\end{array}$ & Physical interpretation & $\begin{array}{l}\text { Typical } \\
\text { value }\end{array}$ & $\begin{array}{l}\text { Value } \\
\text { used }\end{array}$ \\
\hline$H^{*}$ & $4 \times 10^{-4} \mathrm{~cm}$ & $P_{0}=P_{0}^{*} L^{* 4} / \kappa^{*} H^{*}$ & Size of hydration stresses & $4 \times 10^{14}$ & $1 \times 10^{8}$ \\
\hline$L^{*}$ & $2 \times 10^{-3} \mathrm{~cm}$ & $\lambda=\lambda^{*} / H^{*}$ & Length scale of hydration stresses & $3 \times 10^{-5}$ & $5 \times 10^{-3}$ \\
\hline$\kappa^{*}$ & $1 \times 10^{-12} \mathrm{erg}$ & $k_{1}=k_{1}^{*} \mu^{*} L^{* 6} / \kappa^{*} H^{* 4}$ & Osmosis-driven flux & $3 \times 10^{8}$ & $1 \times 10^{2}$ \\
\hline$P_{0}^{*}$ & $1 \times 10^{10} \mathrm{erg} \mathrm{cm}^{-3}$ & $k_{2}=k_{2}^{*} \mu^{*} L^{* 2} / H^{* 3}$ & Pressure-driven flux & $6 \times 10^{-6}$ & $1 \times 10^{-2}$ \\
\hline$\lambda^{*}$ & $1 \times 10^{-8} \mathrm{~cm}$ & $c_{\text {init }}$ & Initial sugar concentration & $5 \times 10^{-3}$ & $1 \times 10^{-2}$ \\
\hline$\rho^{*}$ & $1 \times 10^{0} \mathrm{~g} \mathrm{~cm}^{-3}$ & & & & \\
\hline$\mu^{*}$ & $1 \times 10^{3} \mathrm{~g} \mathrm{~cm}^{-1} \mathrm{~s}^{-1}$ & & & & \\
\hline$k_{1}^{*}$ & $1 \times 10^{-4} \mathrm{~cm} \mathrm{~s}^{-1}$ & $\operatorname{Re}=\rho^{*} \kappa^{*} H^{* 5} / \mu^{* 2} L^{* 6}$ & Importance of inertia & $1 \times 10^{-16}$ & $\ll 1$ \\
\hline$k_{2}^{*}$ & $8 \times 10^{-14} \mathrm{erg}^{-1} \mathrm{~cm}^{4} \mathrm{~s}^{-1}$ & $\mathrm{Pe}=\left(D^{*} / L^{* 2}\right)\left(H^{*} / k_{1}^{*} \Delta c\right)$ & Importance of diffusion & $3 \times 10^{-5}$ & $\ll 1$ \\
\hline$D^{*}$ & $1 \times 10^{-5} \mathrm{~cm}^{2} \mathrm{~s}^{-1}$ & $\epsilon=H^{*} / L^{*}$ & Aspect ratio & $2 \times 10^{-1}$ & $\ll 1$ \\
\hline
\end{tabular}

whose aspect ratio has the moderately small value of $H^{*} / L^{*}=$ 0.2 . If the basal diameter of this cap is given by $L^{*}$, then its volume is given by $2 \pi L^{* 3} / 75$. Desiccation experiments have been performed with cells of various sizes, which include fibroblasts [2] and human embryonic kidney cells [30], which have typical volumes of, respectively, 250 and $4200 \mu \mathrm{m}^{3}$. For this range of volumes, the corresponding values for $L^{*}$ range between 10 and $40 \mu \mathrm{m}$. We use the estimates $L^{*}=20 \mu \mathrm{m}$ and $H^{*}=4 \mu \mathrm{m}$ for the horizontal and vertical length scales, respectively.

Recent experiments [2] report that the cell membrane typically remains intact after desiccation and reconstitution provided the cell is initially loaded with trehalose at a concentration that ranges between $2 \times 10^{-4}$ and $5 \times 10^{-4} \mathrm{~mol} \mathrm{~cm}^{-3}$, although smaller concentrations have also been reported [30] to be sufficient. The molar volume of water is given by $\tilde{V}_{w}=18 \mathrm{~cm}^{3} \mathrm{~mol}^{-1}$ and, hence, the molar concentration of pure water is approximately $5.6 \times 10^{-2} \mathrm{~mol} \mathrm{~cm}^{-3}$. It follows that typical trehalose concentrations, which are assumed to range between $2 \times 10^{-4}$ and $5 \times 10^{-4} \mathrm{~mol} \mathrm{~cm}^{-3}$, correspond to volume fractions that range between $3.5 \times 10^{-3}$ and $9 \times 10^{-3}$. We use the value $c_{\text {init }}=10^{-2}$ in our calculations. At such small volume fractions of sugar, the density of the solution is only very slightly different to that of pure water; we therefore assume that the density of the solution is given by $\rho^{*}=1 \mathrm{~g} \mathrm{~cm}^{-3}$.

The physical properties of the solution are estimated using the Williams-Landel-Ferry (WLF) model [31], which has been validated experimentally [32]. In laboratory experiments [2], the cells are dried at temperatures of $4^{\circ} \mathrm{C}$, at which the viscosity of a solution with volume fraction given by $c=0.01$ is approximately $\mu^{*} \approx 1100 \mathrm{~g} \mathrm{~cm}^{-1} \mathrm{~s}^{-1}$.

The hydration forces that act on the membrane are described by [20] to be given by Eq. (4), where the order-of-magnitude values of the amplitude $P_{0}^{*}$ and decay length $\lambda^{*}$ are, respectively, $10^{10} \mathrm{erg} \mathrm{cm}^{-3}$ and $10^{-8} \mathrm{~cm}$. The bending stiffness of a bilipid membrane is given $[3,33,34]$ as $\kappa^{*}=10^{-12}$ erg. Whereas the qualitative nature of the short-range hydration stresses is included in our model, as shown in Table I we use values for the dimensionless parameters $P_{0}$ and $\lambda$ [defined by (6)] that are significantly different from those encountered in experiment. The values that we use are selected for ease of computation; we do not expect that this choice affects the qualitative nature of the results.

The volume flux of water through a unit area of the membrane, measured in $\mathrm{cm} \mathrm{s}^{-1}$, is given by [35]

$$
Q^{*}=L_{p}^{*}\left[p^{*}-\sigma^{*} R^{*} T^{*} \Delta c^{*}\right],
$$

where $L_{p}^{*}$ is the filtration coefficient, $p^{*}$ is the pressure difference across the membrane, $\sigma^{*}$ is the reflection coefficient of solute, $R^{*}$ is the gas constant, and $\Delta c^{*}$ is the difference in molar concentration of solute across the membrane. At low sugar concentrations, the molar sugar concentration is given approximately by the volume fraction $c_{\text {in }}$ of sugar divided by the molar volume of water. Hence, we take

$$
\bar{V}_{w} \Delta c^{*}=c_{\mathrm{out}}-c_{\mathrm{in}}
$$

from which it follows that

$$
Q^{*}=P_{f}^{*}\left[\frac{\bar{V}_{w}^{*}}{\sigma^{*} R^{*} T^{*}} p^{*}+c_{\mathrm{out}}-c_{\mathrm{in}}\right],
$$

where $P_{f}^{*}$ is given by $L_{p}^{*} \sigma^{*} R^{*} T^{*} / \bar{V}_{w}^{*}$ and is known as the osmotic water permeability constant. The reflection coefficient is given by $\sigma^{*}=1$ for a membrane that is impermeable to sugar and, hence, the ratio between the fluxes driven by pressure and by concentration gradients across the membrane is given by $\bar{V}_{w} / R^{*} T^{*}$. This ratio has the numerical value $7.7 \times$ $10^{-10} \mathrm{erg}^{-1} \mathrm{~cm}^{3}$ at temperature $4^{\circ} \mathrm{C}$. Experiments $[35,36]$ have obtained values of $P_{f}^{*}$ for bilipid membranes that range between $10^{-5}$ and $10^{-2} \mathrm{~cm} \mathrm{~s}^{-1}$. Hence, in our expression (20) for the dimensional volume flux, given by

$$
Q^{*}\left(x^{*}\right)=k_{1}^{*}\left(c_{\text {out }}-c_{\text {in }}\right)+k_{2}^{*} p,
$$

appropriate values for $k_{1}^{*}$ and $k_{2}^{*}$ are, respectively, $10^{-4} \mathrm{~cm} \mathrm{~s}^{-1}$ and $8 \times 10^{-14} \mathrm{erg}^{-1} \mathrm{~cm}^{4} \mathrm{~s}^{-1}$. From (7), and as shown in Table I, these imply extremely large and small values for the dimensionless parameters $k_{1}$ and $k_{2}$, respectively. In our calculations we use values for $k_{1}$ and $k_{2}$ that are only moderately large or small, as appropriate, in order to facilitate numerical calculation while preserving the qualitative nature of the results.

As described in the main text, the extremely large ratio between the fluxes driven by pressure and concentration 
differences across the membrane means that, even for moderately large concentration differences, the flow is dominated by the transport of water across the membrane and viscous stresses typically play a negligible role. In these situations it might be reasonable to assume that $k_{2}=0$. However, the concentrations on either side of the membrane will eventually equalize so that $c_{\text {in }} \approx c_{\text {out }}$, and the osmotic flux will at that time be very small. When this concentration difference is $O\left(k_{2} / k_{1}\right)$, it is no longer valid to omit the pressure-driven flow within the vesicle. Our analysis therefore applies to the behavior of the membrane when the concentration difference $c_{\text {in }}-c_{\text {out }}$ is very small. Our use of a much larger value for $k_{2}$ also decreases the sensitivity of the results to the value of $c_{\text {out }}$, and thus facilitates the presentation of the computed results.

In our model we have omitted the effects of advection on the sugar concentration within the vesicle and of the effects of inertia on the fluid flow. The first of these assumptions requires that diffusive transport across the vesicle is much faster than advection. The slowest diffusive time scale is given by $T_{d}^{*}=L^{* 2} / D^{*}$, where $D^{*}$ is the diffusivity of sugar in solution, and this time scale corresponds to horizontal transport beneath the membrane. The diffusivity of trehalose, at the temperatures and (very small) sugar concentrations encountered in experiment, is of the order of $10^{-6} \mathrm{~cm}^{2} \mathrm{~s}^{-1}$. Together with the length scale given by $L^{*}=2 \times 10^{-3} \mathrm{~cm}$, this implies that the slowest diffusive time scale is given by $T_{d}^{*}=4 \mathrm{~s}$. There are two time scales associated with advective transport; one is given by $T_{f}^{*}=H^{*} / Q^{*}$ and corresponds to the flow driven by volume flux through the membrane, while the other is given by $T_{p}^{*}=L^{*} / U^{*}$ and corresponds to flow that is driven by stresses exerted by the membrane.

The time scale of flow through the membrane depends on the concentration difference across it, according to $T_{f}^{*}=$ $H^{*} / k_{1}^{*}\left(c_{\text {in }}-c_{\text {out }}\right)$. Substitution of the above values for $H^{*}$ and $k_{1}^{*}$ yields $T_{f}^{*}=4 /\left(c_{\text {in }}-c_{\text {out }}\right)$ s. Typical values for $c_{\text {in }}$ and $c_{\text {out }}$ are around $5 \times 10^{-3}$ although, as mentioned above, our analysis applies to situations where the difference between these concentrations is very small. It is therefore reasonable to assume that $T_{f}^{*} \gg T_{d}^{*}$. The second advective time scale $T_{p}^{*}$ is given by $L^{*} / U^{*}$. Fluid flow inside the vesicle is driven by pressure gradients across it, according to $U^{*}=p_{0}^{*} H^{* 2} / \mu^{*} L^{*}$ [from (7)], where $p_{0}^{*}$ is a representative pressure scale. It is convenient in our model to derive a pressure scale using the membrane's (known) bending stiffness rather than its (unknown) compression. However, in the main text it is shown that typical values of the dimensionless compression $\Gamma$ of the membrane are around $10^{2}-10^{3}$ and, hence, pressure-driven flow is caused primarily by the membrane's compression rather than its bending stress. The pressure scale that best represents the flow is therefore given by $p^{*}=\Gamma \kappa^{*} H^{*} / L^{* 4}$, which yields a velocity scale that is given by $U^{*} \approx 2 \times 10^{-9} \mathrm{~cm} \mathrm{~s}^{-1}$. The pressure-driven flow time scale $T_{p}^{*}$ is therefore typically of the order $10^{6} \mathrm{~s}$. Both advective time scales $T_{f}^{*}$ and $T_{p}^{*}$ are much longer than the slowest diffusive time scale $T_{d}^{*}$, which justifies the assumption that the sugar solution inside the vesicle remains homogeneous.

As described in the main text, the omission of inertia is valid provided the dimensionless group given by $\mathrm{Re}=$ $\rho^{*} U^{*} H^{* 2} / \mu^{*} L^{*}$ is very small. This may be justified through substitution of the values obtained above for the relevant dimensional quantities, which yields $\operatorname{Re} \sim 10^{-16}$.

\section{APPENDIX B: CALCULATION OF GROWTH RATES OF DISTURBANCES}

As described in Sec. IV, linearized disturbances to the steady state satisfy

$$
\begin{aligned}
\sigma \widehat{h} & =\left(\frac{\bar{h}^{3}}{12} \widehat{p}_{x}\right)_{x}+k_{1} \widehat{c}_{\mathrm{in}}-k_{2} \widehat{p}, \\
\widehat{p} & =\widehat{h}_{x x x x}+\bar{\Gamma} \widehat{h}_{x x}+\widehat{\Gamma} \bar{h}_{x x}+\frac{P_{0} \widehat{h} \exp (-\bar{h} / \lambda)}{\lambda},
\end{aligned}
$$

subject to the constraints

$$
\begin{array}{r}
\bar{c}_{\text {in }} \int_{-1 / 2}^{1 / 2} \widehat{h} d x+\widehat{c}_{\text {in }} \int_{-1 / 2}^{1 / 2} \bar{h} d x=0, \\
\int_{-1 / 2}^{1 / 2} \bar{h}_{x} \widehat{h}_{x} d x=0,
\end{array}
$$

which determine the disturbances $\widehat{c}_{\text {in }}$ to the sugar concentration inside the vesicle and $\widehat{\Gamma}$ to the compression of the membrane. This system of equations may be formulated as a generalized eigenvalue problem for the variables $\widehat{h}, \widehat{p}, \widehat{c}_{\text {in }}$, and $\widehat{\Gamma}$. However, we found that this problem is very poorly conditioned, and that better resolution of the eigenvalues is possible by instead eliminating $\widehat{p}, \widehat{c}_{\text {in }}$, and $\widehat{\Gamma}$. To do this, we first use the integral constraints to eliminate $\widehat{c}_{\text {in }}$ and $\widehat{\Gamma}$ in favor of $\widehat{h}$. The first integral constraint implies that

$$
\widehat{c}_{\text {in }}=-\frac{\int_{-1 / 2}^{1 / 2} \widehat{h} d x}{\int_{-1 / 2}^{1 / 2} \bar{h} d x} \bar{c}_{\text {in }},
$$

whereas the second constraint may be integrated by parts to obtain

$$
\int_{-1 / 2}^{1 / 2} \bar{h}_{x x} \widehat{h} d x=0 .
$$

[The boundary terms vanish by virtue of the boundary conditions (39) prescribed to the eigenmode.] We may eliminate $\widehat{\Gamma}$ by multiplying both sides of (B1a) by $\bar{h}_{x x}$ and integrating over the length of the membrane, which yields

$$
\begin{aligned}
& \sigma \int_{-1 / 2}^{1 / 2} \bar{h}_{x x} \widehat{h} d x \\
& \quad=\sigma \int_{-1 / 2}^{1 / 2} \bar{h}_{x x}\left[\left(\frac{\bar{h}^{3}}{12} \widehat{p}_{x}\right)_{x}+k_{1} \widehat{c}_{\mathrm{in}}-k_{2} \widehat{p}\right] d x=0 .
\end{aligned}
$$

We note that this introduces a spurious solution, for which $\int_{-1 / 2}^{1 / 2} \bar{h}_{x x} \widehat{h} d x \neq 0$ and $\sigma=0$, which must be discarded when the eigenmodes are calculated using this method. However, the computed value of $\sigma$ for this eigenmode provides some heuristic indication of the accuracy of the other calculated eigenvalues. We substitute for $\widehat{p}$ using (B1b) to obtain

$$
\widehat{\Gamma}=-\frac{\tilde{\Gamma}}{\Gamma_{D}},
$$


where

$$
\begin{aligned}
\tilde{\Gamma}= & \int_{-1 / 2}^{1 / 2} \bar{h}_{x x}\left\{\left[\frac { \overline { h } ^ { 3 } } { 1 2 } \left(\widehat{h}_{x x x x x}+\bar{\Gamma} \widehat{h}_{x x x}\right.\right.\right. \\
& \left.\left.+\frac{P_{0} \widehat{h}_{x}}{\lambda} e^{-\bar{h} / \lambda}-\frac{P_{0} \widehat{h} \bar{h}_{x}}{\lambda^{2}} e^{-\bar{h} / \lambda}\right)\right]_{x} \\
& \left.+k_{1} \widehat{c}_{\text {in }}-k_{2}\left(\bar{\Gamma} \widehat{h}_{x x}+\widehat{h}_{x x x x}+\frac{P_{0} \widehat{h}}{\lambda} e^{-\bar{h} / \lambda}\right)\right\} d x,
\end{aligned}
$$

and

$$
\Gamma_{D}=\int_{-1 / 2}^{1 / 2} \bar{h}_{x x}\left[\left(\frac{\bar{h}^{3} \bar{h}_{x x x}}{12}\right)_{x}-k_{2} \bar{h}_{x x}\right] d x
$$

After elimination of $\widehat{c}_{\text {in }}$ and $\widehat{\Gamma}$ using (B2) and (B3), the only disturbance variable that remains is $\widehat{h}$, and the steadystate variables $\bar{h}, \bar{\Gamma}$, and $\bar{c}_{\text {in }}$ may be calculated as described in Sec. III. We discretize this simplified problem using a second-order finite-difference scheme on an equispaced grid of between 100 and 500 points. The integral constraints are implemented using Simpson's rule. We find that roundoff error obscures the eigenmodes of interest when more than around 400 points is used, and that, for smaller numbers of points, the eigenvalues typically converge only to within at most two significant figures. As described in the main text, we therefore calculate solutions to this eigenvalue problem using the high-accuracy routines of the software package AUTO-07P to ensure that the eigenmodes are properly resolved.
[1] J. H. Crowe, F. A. Hoekstra, and L. M. Crowe, Annu. Rev. Physiol. 54, 579 (1992).

[2] T. Chen, J. P. Acker, A. Eroglu, S. Cheley, H. Bayley, A. Fowler, and M. Toner, Cryobiology 43, 168 (2001).

[3] W. Helfrich, Z. Naturforsch. C 28, 693 (1973).

[4] A. Aksan, D. Irimia, X. He, and M. Toner, J. Appl. Phys. 99, 064703 (2006).

[5] N. Chakraborty, A. Chang, H. Elmoazzen, M. A. Menze, S. C. Hand, and M. Toner, Ann. Biomed. Eng. 39, 1582 (2011).

[6] R. Lipowsky, M. Brinkmann, R. Dimova, T. Franke, J. Kierfeld, and X. Zhang, J. Phys. Condens. Matter 17, S537 (2005).

[7] U. Seifert and R. Lipowsky, Phys. Rev. A 42, 4768 (1990).

[8] J. Zhang, S. Das, and Q. Du, J. Comput. Phys. 228, 7837 (2009).

[9] P. S. Swain and D. Andelman, Langmuir 15, 8902 (1999).

[10] U. Seifert, K. Berndl, and R. Lipowsky, Phys. Rev. A 44, 1182 (1991).

[11] H. Jian-Guo and O.-Y. Zhong-Can, Phys. Rev. E 41, 461 (1993).

[12] I. Cantat and C. Misbah, Phys. Rev. Lett. 83, 235 (1999).

[13] D. Salac and M. Miksis, J. Comput. Phys. 230, 8192 (2011).

[14] Q. Zhang and T. A. Witten, Phys. Rev. E 76, 041608 (2007).

[15] M. Ortiz and G. Gioia, J. Mech. Phys. Solids 42, 531 (1994).

[16] P. Castaing, L. Lemoine, and A. Gourdenne, Compos. Struct. 30, 217 (1995); 30, 223 (1995).

[17] J. N. Israelachvili and P. M. McGuiggan, Science 241, 795 (1998).

[18] A. Oron, S. H. Davis, and S. G. Bankoff, Rev. Mod. Phys. 69, 931 (1997).
[19] U. Seifert, Eur. Phys. J. B 8, 405 (1999).

[20] S. Leikin, V. A. Parsegian, D. C. Rau, and R. P. Rand, Annu. Rev. Phys. Chem. 44, 369 (1993).

[21] J. Faraudo and F. Bresme, Phys. Rev. Lett. 94, 077802 (2005).

[22] L. Lu and M. L. Berkowitz, Mol. Phys. 104, 3607 (2006).

[23] M. J. Miksis and S. H. Davis, J. Fluid Mech. 273, 125 (1994).

[24] E. J. Doedel, H. B. Keller, and Kernévez, Int. J. Bifurcation Chaos Appl. Sci. Eng. 1, 493 (1991); 1, 745 (1991).

[25] H. B. Keller, Numerical Methods for Two-Point Boundary-Value Problems (Dover, New York, 1992).

[26] N. M. Ribe, M. Habibi, and D. Bonn, Phys. Fluids 18, 084102 (2006).

[27] N. M. Ribe, J. R. Lister, and S. Chiu-Webster, Phys. Fluids 18, 124105 (2006).

[28] H. B. van Lengerich, M. J. Vogel, and P. H. Steen, Physica D 238, 531 (2009).

[29] Y. Cao and J. W. Hutchinson, Proc. R. Soc. A 468, 94 (2012).

[30] N. Guo, I. Puhlev, D. R. Brown, J. Mansbridge, and F. Levine, Nat. Biotechnol. 18, 168 (2000).

[31] M. L. Williams, R. F. Landel, and J. D. Ferry, J. Am. Chem. Soc. 77, 3701 (1955).

[32] X. He, A. Fowler, and M. Toner, J. Appl. Phys. 100, 074702 (2006).

[33] L. Bo and R. E. Waugh, Biophys. J. 55, 509 (1989).

[34] U. Seifert, Adv. Phys. 46, 13 (1997).

[35] R. Fettiplace and D. A. Haydon, Physiol. Rev. 60, 510 (1980).

[36] D. W. Deamer and J. Bramhall, Chem. Phys. Lipids 40, 167 (1986). 